\title{
A reproducible approach to high-throughput biological data acquisition and integration
}

Daniela Boernigen, Yo S Moon, Gholamali Rahnavard, Levi Waldron, Lauren Mclver, Afrah Shafquat, Eric Franzosa, Larissa Miropolsky, Christopher Sweeney, Xochitl C Morgan, Wendy Garrett, Curtis Huttenhower

Modern biological research requires rapid, complex, and reproducible integration of multiple experimental results generated both internally and externally (e.g. from public repositories). Although large systematic meta-analyses are among the most effective approaches both for clinical biomarker discovery and for computational inference of biomolecular mechanisms, identifying, acquiring, and integrating relevant experimental results from multiple sources for a given study can be time-consuming and error-prone. To enable efficient and reproducible integration of diverse experimental results, we developed a novel approach for standardized acquisition and analysis of high-throughput and heterogeneous biological data. This allowed, first, novel biomolecular network reconstruction in human prostate cancer, which correctly recovered and extended the NFKB signaling pathway. Next, we investigated host-microbiome interactions. In less than an hour of analysis time, the system retrieved data and integrated six germ-free murine intestinal gene expression datasets to identify the genes most influenced by the gut microbiota, which comprised a set of immune-response and carbohydrate metabolism processes. Finally, we constructed integrated functional interaction networks to compare connectivity of peptide secretion pathways in the model organisms Escherichia coli, Bacillus subtilis, and Pseudomonas aeruginosa. 


\section{A reproducible approach to high-throughput biological data acquisition and integration}

4 Daniela Börnigen ${ }^{127^{*}}$, Yo Sup Moon ${ }^{1 *}$, Gholamali Rahnavard ${ }^{12}$, Levi Waldron ${ }^{18}$, Lauren McIver ${ }^{1}$,

5 Afrah Shafquat ${ }^{1}$, Eric A. Franzosa ${ }^{12}$, Larissa Miropolsky ${ }^{1}$, Christopher Sweeney ${ }^{3}$ Xochitl C.

6 Morgan $^{12 *}$, Wendy S. Garrett ${ }^{2456 *}$, Curtis Huttenhower ${ }^{12 \#}$

$7 \quad{ }^{1}$ Biostatistics Department, Harvard School of Public Health, Boston, MA 02115, USA.

$8 \quad 2$ The Broad Institute of MIT and Harvard, Cambridge, MA 02142, USA.

$9 \quad 3$ Dana-Farber Cancer Institute, Boston, MA 02115, USA.

$10{ }^{4}$ Department of Immunology and Infectious Diseases, Harvard School of Public Health, Boston, MA 1102115 , USA.

$12{ }^{5}$ Department of Medicine, Harvard Medical School, Boston, MA 02115, USA.

$13{ }^{6}$ Department of Medical Oncology, Dana-Farber Cancer Institute, Boston, MA 02115, USA.

${ }^{7}$ Present Address: Department of Human Genetics, the University of Chicago, Chicago, IL 60637, USA.

${ }^{8}$ City University of New York School of Public Health, Hunter College, New York, NY 10035, USA.

* Equally contributed.

\# To whom correspondence should be addressed. Tel: +1-617-432-4912; Fax: +1-617-432-5619;

Email: chuttenh@hsph.harvard.edu

\section{ABSTRACT}

Modern biological research requires rapid, complex, and reproducible integration of multiple experimental results generated both internally and externally (e.g. from public repositories). Although large systematic meta-analyses are among the most effective approaches both for clinical biomarker discovery and for computational inference of biomolecular mechanisms, identifying, acquiring, and integrating relevant experimental results from multiple sources for a given study can be timeconsuming and error-prone.

To enable efficient and reproducible integration of diverse experimental results, we developed a novel approach for standardized acquisition and analysis of high-throughput and heterogeneous biological data. This allowed, first, novel biomolecular network reconstruction in human prostate cancer, which correctly recovered and extended the NFKB signaling pathway. Next, we investigated host-microbiome interactions. In less than an hour of analysis time, the system retrieved data and integrated six germfree murine intestinal gene expression datasets to identify the genes most influenced by the gut microbiota, which comprised a set of immune-response and carbohydrate metabolism processes. Finally, we constructed integrated functional interaction networks to compare connectivity of peptide secretion pathways in the model organisms Escherichia coli, Bacillus subtilis, and Pseudomonas aeruginosa. 
39 We have provided a publicly-available implementation of this approach, ARepA (Automated 40 Repository Acquisition, http://huttenhower.sph.harvard.edu/arepa), which allows configurable access 41 for any organism(s) to a range of high-throughput data sources (currently GEO, IntAct, BioGRID, 42 RegulonDB, STRING, Bacteriome, and MPIDB). Users can retrieve raw or processed data and 43 metadata, normalize values and formats, and automatically standardize subsequent processing to ensure 44 reproducibility. ARepA currently provides $>630 \mathrm{~K}$ interactions and $>15 \mathrm{~K}$ gene expression data sets 45 (>530K samples) for six model organisms, accompanied by corresponding metadata and derived

46

47

48

49

50

51

52

53

54

55

56

57

58

59

60

61

62

63

64

65

66

67

68

69

70

71

72

73

74

75

76

77

\section{INTRODUCTION}

The challenges of "big data" handling in modern biology have been highlighted repeatedly in recent publications (Lynch 2008; Marx 2013; Staff 2011). The need for reproducible analyses of individual and integrated genome-scale data has resulted both in significant progress (Chan et al. 2008; Waldron et al. 2012; Wirapati et al. 2008) and in substantial setbacks, such as the widely-publicized failed colon and ovarian cancer clinical trials (Dressman et al. 2007; Garman et al. 2008; Potti et al. 2006; Samuel Reich 2011). Reproducibility in experimental biology is enforced by a combination of best practices and technical aids built up over decades of research: standardized protocols, reagents, recording practices, and equipment that ease the organizational burden necessary for high-quality research. Although parallel best practices have begun to be widely available for the equivalent processes in bioinformatic and data science investigations (Charaniya et al. 2008; Noble 2009; Wilson et al. 2014), they are not yet widely supported by standardized methods or computational environments enforcing reproducibility, which we develop here.

Reproducibly executing any single high-throughput biological study can be challenging, while reproducibly integrating multiple large-scale data sources presents an even greater hurdle. Metaanalysis of multiple genome-scale studies of the same biological system has proven to be a fruitful route for successful translational research, with over 500 such meta-analyses of human tumor gene expression alone now published (Chan et al. 2008; Wirapati et al. 2008). However, retrieving, standardizing, and curating such data is nontrivial, time-consuming, and error-prone. Integration of heterogeneous data can be an even more onerous process. For example, GEO (Barrett et al. 2011) and the BioGRID (Stark et al. 2011) each have over 2,000 citations as individual data sources, but fewer than 100 publications cite both (Warde-Farley et al. 2010). This lack of automatable systems for largescale biological data handling and retrieval means that reliably incorporating such data into an analytical project is precluded without extensive computational expertise, and even in the best case, it is prone to error and challenging to reproduce (Gentleman 2005; Ioannidis et al. 2009). This is particularly true when incorporating data from more than one source or type, or when linking data from public repositories to additional datasets from one or more individual labs. An example application, such as the identification of disease biomarkers, may involve establishing candidate markers by synthesis of published gene expression data, linking promising candidate markers to proteomic 
78 experiments, establishing putative biological mechanisms through databases of regulatory and protein 79 interactions, and finally validating only the best candidates manually in the lab (Lu et al. 2005; Rifai et 80 al. 2006; van 't Veer et al. 2002). The advantages of such holistic approaches are obvious, but greatly 81 expand the complexity of analysis.

82 Data retrieval systems have recently been developed that facilitate this process for consistent, largescale access to individual 'omics repositories. The Gene Expression Omnibus (GEO) (Barrett et al. 2011; Barrett et al. 2013) and ArrayExpress (Rustici et al. 2013), for example, represent two of the largest functional genomics resources for transcriptional data, and both feature elegant web-based query systems (e.g. the Gene Expression Atlas (Kapushesky et al. 2012)) and programmatic access (e.g. GEOQuery (Davis \& Meltzer 2007)). Similarly, molecular interaction databases such as IntAct (Kerrien et al. 2012; Orchard et al. 2014) and the Biological General Repository for Interaction Datasets (BioGRID) (Chatr-Aryamontri et al. 2013; Stark et al. 2011) typically provide access to selected subsets of protein-protein interaction networks by way of taxonomic, technological, or temporal metadata. Each of these interfaces is database-specific, although they provide their data downloadable in the PSI-MI standard format; in general, expression data retrieved programmatically in $\mathrm{R}$ must be manually converted and combined with interaction data. Although some systems such as DAS (Dowell et al. 2001) provide a uniform interface for multiple 'omic data types, accessing data from most public repositories presents a major challenge in the compatibility of the resulting data formats, mapping between different gene identifier conventions, and the curation of inconsistentlyannotated experiments. Manual parsing and conversion of experimental attributes and values is tedious and error-prone (Baggerly \& Coombes 2009), and the large number of normalization and preprocessing methods in use make comparison of different existing studies difficult or even impossible (Sims 2009). This suggests the need for a unified approach capable of automatically retrieving and reproducibly processing data from multiple different 'omics repositories.

Previous related efforts for reproducible research include 1) general workflow systems for broad bioinformatic analysis that can be tuned for data retrieval, 2) tools enabling programmatic access to public databases, and 3) curated alternatives to the largest public experimental databases. General workflow systems, such as Kepler (Barseghian et al. 2010) and Taverna (Hull et al. 2006), facilitate the construction of reproducible programmatic workflows. In many cases they include programmatic access to biological databases, but subsequent data and metadata format harmonization must be implemented separately by the user. Tools that allow programmatic access to public databases, such the specialized interfaces provided by GEOquery (Davis \& Meltzer 2007) and Ensembl BioMarts (Kinsella et al. 2011), are among the most common. GEOquery is specific to automated queries and downloads from one particular database, and while queries can be written across multiple BioMart databases, even the latter tool includes no facilities for normalization, integration, or meta-analysis of the resulting independent query responses. Finally, curated alternative data management and analysis platforms, such as EZArray (Zhu \& Xu 2008), BxArrays ${ }^{\mathrm{TM}}$, and ONCOMINE (Rhodes et al. 2004), provide web interfaces in which a user may perform only pre-selected analyses of the provided gene expression datasets. Another open data management and access platform, inSilicoDB (Taminau et al. 
117 2011), allows programmatic download of gene expression data curated by maintainers or contributors 118 from the community, although users may only retrieve one experiment at a time. None of these tools 119 facilitate bulk download of 'omics data from multiple sources for efficient processing on local 120 computing resources, and most enable only per-repository data acquisition. A logical next step for 121 large scale, multi'omic data acquisition for meta-analysis and heterogeneous data integration is again a 122 batch-driven data acquisition system spanning multiple sources.

123 Motivated by this need to access and integrate diverse sources of experimental results, we developed

124

125

126

127

128

129

130

131

132

133

134

135

136

137

138

139

140

141

142

143

144

145

146

147

148

149

150

151

152

153

154

155 an approach for reproducible processing of high-throughput and heterogeneous data. The system, implemented as the ARepA (Automated Repository Acquisition) software environment, enabled us to rapidly and systematically retrieve, integrate, and analyze large 'omics datasets from multiple sources in a consistent and robust fashion. Here, we first undertook a study of the NFKB signaling pathway in the context of human prostate cancer by integrating ten tissue-specific human expression datasets across six platforms. This not only recovered but extended the currently known molecular network. Next, due to burgeoning recent interest in microbiome studies and the considerable knowledge gaps regarding host processes are dependent upon the gut microbiota, we investigated transcripts that were differentially expressed in the germ-free mouse colon, identifying and integrating six gene expression datasets. PPAR-alpha, RXR-alpha, and several other immune- and carbohydrate metabolism-related genes were detected as differentially expressed in this gene expression meta-analysis. Finally, we integrated protein-protein interactions, gene expression, and regulatory networks for three very diverse model bacteria (Bacillus subtilis, which is Gram-positive; Escherichia coli, which is Gram-negative, and Pseudomonas aeruginosa, which is Gram-negative and pathogenic), correctly identifying differentially-conserved peptide secretion system pathways.

Each of these three studies required rapid, reproducible, and diverse data collection and integration, and thus demonstrates the utility of ARepA for any lab wishing to combine its own data with public data repositories. We provide an implementation of ARepA that can access 'omics data from multiple data sources in a consistent and robust fashion. Furthermore, it can be configured to process data only from certain sources, of specified types, or from particular organisms of interest, depending on the user's interest and requirements. Currently, it supports seven repositories: the Gene Expression Omnibus (GEO) (Barrett et al. 2011; Barrett et al. 2013), IntAct (Kerrien et al. 2012; Orchard et al. 2014), BioGRID (Stark et al. 2011), RegulonDB (Gama-Castro et al. 2011), STRING (Szklarczyk et al. 2011), Bacteriome (Su et al. 2008), and MPIDB (Goll et al. 2008) (Table 1), from which data are retrieved, processed and standardized for any organism of interest. At present, this produces more than $2 \mathrm{M}$ interactions from $\sim 600 \mathrm{~K}$ physical interactions (Bacteriome, BioGRID, Intact, and MPIDB), $\sim 4 \mathrm{~K}$ regulatory interactions (RegulonDB) and 1.5M functional associations (STRING), as well as more than 2.7K expression data sets covering more than $800 \mathrm{~K}$ samples (GEO). When retrieving data from six model organism, ARepA currently produces $>630 \mathrm{~K}$ interactions and $>15 \mathrm{~K}$ gene expression data sets ( $>530 \mathrm{~K}$ samples), accompanied by corresponding metadata and derived molecular networks (Table 2, Figure 1). 


\section{MATERIAL AND METHODS}

\section{Case studies}

159

160

161

162

163

164

165

166

167

168

169

170

171

172

173

174

175

176

177

178

179

180

181

182

183

184

185

186

187

188

189

190

191

Human prostate cancer data retrieval and molecular network integration

We screened ARepA's GEO metadata database for human prostate cancer and prostate tissue conditions matching "prostate cancer" in human gene expression profile studies with at least 6 samples each, identifying ten relevant datasets from six different platforms (Affymetrix, Agilent, and CNIO, Supplementary Table 1). Identifying, processing, and standardizing these datasets in ARepA, accompanied by computing co-expression networks, required $<1$ hour running time in total (on a 2.9 $\mathrm{GHz}$ Intel Core 17 16GB machine). These data were then meta-analyzed to a single prostate cancer specific functional network using unsupervised data integration averaging across normalized coexpression values (z-scores) (Huttenhower et al. 2009) (Supplementary Text 1). Next, we used this integrated network to predict genes highly functionally related to $\mathrm{NF} \kappa \mathrm{B}$ gene family $(\mathrm{NF} \kappa \mathrm{B} 1, \mathrm{NF} \kappa \mathrm{B} 2$, RELA, RELB, REL) in prostate cancer by extracting a high-confidence subgraph based on the probabilistic graph search algorithm as described in (Huttenhower et al. 2009; Myers et al. 2005). This starts from a user-defined set of query genes and identifies $k$ additional neighbours in the network that are connected with high confidence to the original query genes(Huttenhower et al. 2008). Here, we defined a query gene set of twelve genes from the NFkB signaling pathway in BioCarta (Supplementary Table 4) and a neighbourhood size of $k=10$, resulting in a high-confidence NFkB signaling network containing 22 genes in total as illustrated in Figure 2.

\section{Murine expression data retrieval and meta-analysis}

We conducted a differential expression meta-analysis of genes and pathways up- or down-regulated in the germ-free (without microbes) murine gut (Supplementary Figure 1, Supplementary Text 1). We used six case-control datasets containing intestinal tissue and germ-free versus wild type comparisons, identified through ARepA's metadata screen by matching "germ-free", "wild type", and "intestinal tract" in mouse gene expression profiles with at least four samples each (Supplementary Table 2). This again required $<1$ hour total running time (on a $2.9 \mathrm{GHz}$ Intel Core i7 $16 \mathrm{~GB}$ machine). To integrate the resulting ARepA R data files, we first computed log fold changes and confidence intervals $(2.5 \%$ and $97.5 \%)$ for all genes between germ-free gut and wild type gut within each dataset using the $\mathrm{R} /$ limma package (Smith 2005), resulting in $~ 3,600$ differentially expressed genes. Next, we performed a meta-analysis using the $\mathrm{R} /$ metafor package (Viechtbauer 2010) on the six datasets by applying a random-effects model on the fold changes with default options, fitting the model with the restricted maximum-likelihood estimator (REML). The false discovery rate was controlled by the BenjaminiHochberg method (Benjamini 1995). We finally tested all genes and their resulting meta-p-values for gene set enrichment (Subramanian et al. 2005) in KEGG and BioCarta pathways. This resulted in two pathways that were significantly enriched for upregulated genes under germ-free conditions (using 
192 1,000 permutations, Figure 3), while 15 pathways were enriched for downregulated genes 193 (Supplementary Table 3).

194

195

196

197

198

199

200

201

202

203

204

205

206

207

208

209

210

211

212

213

214

215

216

217

218

219

220

221

222

223

224

225

226

227

\section{Data integration for bacterial model organisms}

We retrieved all gene expression data (GEO) and gene interaction data (IntAct, MPIDB, RegulonDB, Bacteriome and STRING, Table 2A) from ARepA for Bacillus subtilis, Escherichia coli and Pseudomonas aeruginosa. These were processed as above and standardized onto a shared gene identifier (UniRef90). Within each bacterium, these data were integrated into a species-specific functional network using an unsupervised data integration averaging across normalized co-expression values (z-scores) (Huttenhower et al. 2009) and extracting a high-confidence subgraph based on the probabilistic graph search algorithm (Huttenhower et al. 2009; Myers et al. 2005) (Supplementary Text 1). This query (Huttenhower et al. 2008) started from a user-defined set of genes of the sec and tat genes and genes from the Type I, Type II, Type III, Type V, and Type VI secretion systems (Supplementary Table 4) and used a neighbourhood size of $k=5$ for each subgraph extraction. As Gram-positive bacteria use only the general secretory (sec) pathway and the tat pathway, we used two tat genes and nine sec genes as the query gene set in B. subtilis. In contrast, E. coli, a Gram-negative bacterium, employs an additional secretion system (Type II secretion system) and has one type I gene (TolC); thus, we used four tat genes, twelve sec genes, one type I gene, and twelve type II genes as a query gene set in E. coli. P. aeruginosa, another Gram-negative bacterium, additionally utilizes the type I, type III, type V, and type VI secretion systems, so we defined our query gene set of three tat genes, nine sec genes, two type I genes, eleven type II genes, twelve type III genes, one type V gene, and eight type VI genes for retrieving a species-specific high-confidence network in $P$. aeruginosa. We recovered networks of 16 genes for $B$. subtilis (Figure 4A), 34 genes for E. coli (Figure 4B), and 49 genes for $P$. aeruginosa (Figure 4C), which showed clustering of secretion types conserved across species.

\section{Implementation}

ARepA is designed around "dedicated" and "shared" processing modules. Dedicated modules download and process raw data from individual public repositories, while shared modules process the resulting data into standardized formats and aid key steps common to multiple dedicated modules. This enables ARepA to customize the unique aspects of individual public repositories' interfaces while sharing consistent information across all repositories, such as systematic identifiers for taxonomy, genes, and metadata.

Each repository in ARepA is implemented as one dedicated module, all of which share a similar design template. The main shared module typically executed in any dedicated module is the GeneMapper, which installs and initializes the BridgeDB package (BatchMapper command line tool) (van Iersel et al. 2010) to map gene identifier consistently across all data types after data retrieval. Later in each repository's processing steps, the shared Package module is executed to export all output data (such as 
228 expression datasets, networks, and metadata) into $\mathrm{R}$ data files, in addition to tab-delimited text, 229 enabling an easy access to the data for downstream analysis.

230 ARepA is implemented in Python and is based on the SCons tool for dependency-driven modular 231 computing. SCons ensures that data processing is efficient (i.e. on an as-needed basis), modular 232 (necessary processing steps for each repository and data product are isolated), and simple (a single 233 command is used to retrieve and integrate all requested data). Therefore, new data are only generated 234 when necessary due to changes in an upstream element, such as new data versions or availability. 235 These changes may include updates to remote data or metadata (e.g. file size or timestamp changes), 236 modifications to the requested processing steps (scripts or configuration files), or changes to the 237 ARepA configuration itself.

\section{ARepA filesystem and configuration}

240 ARepA's implementation consists of a filesystem hierarchy in which the top level coordinates overall 241 execution and contains shared code and documentation, and each subdirectory contains the custom processes necessary to access a specific data repository. Currently, ARepA houses seven sub-modules for its default repositories: GEO (Barrett et al. 2011), IntAct (Kerrien et al. 2012), BioGRID (Stark et al. 2011), Bacteriome (Su et al. 2008), RegulonDB (Gama-Castro et al. 2011), MPIDB (Goll et al. 2008), and STRING (Szklarczyk et al. 2011). Each module in ARepA contains two groups of persistent elements: (1) driver scripts (SCons-based SConstruct and SConscript files) and (2) module-

247

248

249

250

251

252

253

254

255

256

257

258

259

260

261

262 specific directories. Each driver script launches all processes required to subsequently launch child processes in submodules. Module-specific directories are organized consistently to include: (1) $s r c$ folders with module-specific source for data processing, (2) etc folders contain all configurable and user-defined information of the module, (3) tmp folders store intermediate downloaded files before processing and standardization, (4) doc folders contain documentation specific to module, and (5) data folders store the output of the final processed and standardized data.

ARepA includes a global configuration file that optionally limits the processing of all data to a subset of organisms/clades from the NCBI taxonomy (Sayers et al. 2009). Additionally, module-specific configuration files may exist to provide more specific configuration settings (see below). Notably, the GEO module has configuration files to change the type of datasets retrieved (GSE/GDS/GPL/GSM), how microarray values are normalized, which standard gene identifiers are used, the addition of manually curated metadata for specific datasets, and regular expression searches of available data. Due to ARepA's modular design, each repository's data can be handled independently by its corresponding driver script without the need of processing all available repositories, while also incorporating global configuration information from higher levels in the hierarchy.

\section{Configuration files and formats}


263 Procedural configuration files allow different combinations of normalization or processing to be 264 carried out on different ARepA data subsets. For example, the default configuration file for a pre265 processing step in GEO contains the string "affy::rma", which instructs ARepA to normalize 266 expression data with the Robust Multi-array Average (RMA) method (Irizarry et al. 2003). Another 267 option is the MAS5 method (Affymetrix 2002), which can be called with the "affy::mas5" string; 268 complete documentation is provided in the ARepA online manual. Override configuration files, 269 conversely, provide additional metadata (typically manually curated) to be associated with downloaded 270 data. For instance, providing a manually curated override metadata file for the GEO dataset GSE10183 271 in the corresponding configuration directory (GEO/etc/manual_curation/GSE10183.txt) will instruct 272 ARepA to bypass the default metadata pipeline and use the override configuration file as a gold 273 standard instead.

274 Top-level procedural configuration allows the user to configure the taxonomy and gene identifier 275 format of interest. Repository-specific procedural configuration includes the specification of 276 preprocessing functions, regular expression searches for gene mapping, and including/excluding 277 datasets. Repository-specific override configuration allows the incorporation of user-curated metadata 278 and gene identifier maps along with automatically generated metadata.

279

280

281

282

283

284

285

286

287

288

289

290

291

292

293

294

295

296

297

298

299

\section{Data and metadata}

ARepA provides expression, gene interaction data, and metadata as output. ARepA handles three expression data file types: raw, mapped, and normalized. Raw expression data are the default as acquired from the repository, while mapped data include probe IDs assigned to gene names. Finally, normalized expression data are the output of a process consisting of a z-score transformation and missing value imputation as implemented by Sleipnir (Huttenhower et al. 2008). Here, raw values are normalized by transforming the vector of expression values for each gene to z-scores (i.e. mean zero and standard deviation one). Subsequently, missing values are imputed with a weighted average of the corresponding values in their 10 nearest neighbors (weighted by relative Euclidean distance). To aid in assessing data quality, ARepA generates a standardised Quality Control (QC) report using the Bioconductor package arrayQualityMetrics (Kauffmann et al. 2009).

ARepA's reliance on the SCons build system for dependency-driven processing ensures that remote data and metadata are only re-downloaded when changed. There are two corresponding types of interaction data-raw and mapped-which contain default gene pairs and mapped gene pairs, respectively. Currently, gene expression data is retrieved uniquely by GEO, while interaction data is available for all repositories. Corresponding metadata and a co-expression network (Huttenhower et al. 2008) are also generated which are summarized in a portable $\mathrm{R}$ data file. ARepA generates interaction and regulatory networks with corresponding metadata information for all internal modules.

ARepA's metadata model generalizes to accommodate essentially any source repository information and comprises an unordered collection of nested key-value pairs. Each final data file is associated with exactly one metadata file, which includes all information that is available about a dataset through 
300

301

302

303

304

305

306

307

308

309

310

311

312

313

314

315

316

317

318

319

320

321

322

323

324

325

326

327

328

329

330

331

332

333

334

335

336

337

curation or each repository's automated retrieval. Standardized metadata across repositories include each dataset's taxonomic identification, publication identifier, platform identifier, experimental information (see Table 1, Supplementary Text 2) and provenance pertaining to ARepA's internal processes, such as a log of each command run, its version, MD5 checksums, and the success status of the gene standardization for a specific dataset. If requested, curated metadata can be combined with generated metadata (e.g. when a table of per-sample detailed clinical covariates is available).

\section{Reproducible data processing}

Although ARepA will operate using default configuration parameters out-of-the-box, it provides four main input interfaces through which its behavior can be modified: global taxonomic targeting, global gene identifier standardization, per-repository dataset ID restriction, and curated metadata. To ensure standardized data retrieval, ARepA contains global configuration files which regulate the kind of data that will be produced in each repository. Either all data from every repository can be retrieved, or the species for which data is requested can be limited by NCBI Taxonomy identifier or taxon name. Likewise, any target gene identifier formatted supported by GeneMapper can be specified to ensure standardized output data across all repositories.

Each repository has additional custom configuration parameters that specify which datasets are to be included or excluded from processing. These are specified using the unique dataset identifiers for each repository, e.g. GSD or GSE dataset IDs for GEO. Finally, metadata available for each dataset from any repository is included automatically, but all repositories also allow curated metadata to be automatically merged with these provided metadata. Curated metadata are automatically detected if provided in a standardized location within each repository module and, like repository-provided metadata, can include key-value pairs in pre-specified templated formats (e.g. numerical keys, enumerated values, free text, and so forth). This allows for the construction of truly customized highthroughput meta-analytic studies.

ARepA's main outputs are one or more data file(s) (text format) and a metadata file (Python pickle format) per dataset (Supplementary Text 2); this is Python's native serialization format and is efficient to use and query for rapid database joins and searches. The output data consists of 1) a tab-delimited text matrix when appropriate (e.g. gene expression) of the raw data as downloaded from the repository, the processed data, and the standardized data after gene identifier mapping; 2) a tab-delimited network edge list of physical interactions, regulatory interactions, or co-expression from normalized and standardized data; and 3) an $\mathrm{R}$ data file integrating all processed and standardized output and metadata files for each expression data set as a Bioconductor ExpressionSet in one $\mathrm{R}$ file, containing experimental data (experimentData: "Experimenter name", "Laboratory", "Contact information", "Title", "PMIDs", "Abstract", and "notes"), annotation (annotation: platform GPL identifier), phenotypic data (phenoData: per-condition metadata (GSE only), as obtained from GEO by default or the custom curated per-sample metadata if provided at runtime), and gene expression data (exprs: expression data with mapped (unmapped for GDS) probeset identifiers). All raw, processed and standardized output data and metadata files are stored in one directory per dataset within the 
corresponding repository's data directory, and custom processing modules can be included in any repository to produce additional outputs (or modify the default outputs). Each repository's data output directory consists of a structure of subdirectories, organizing the output data into dataset-, taxa- and publication-specific subfolders that contain their corresponding output files (as described above) for an easy and intuitive access to specific output data of interest.

\section{Adding repository modules}

ARepA's modular design allows the seamless integration of custom submodules provided by the user. A user can extend ARepA to include new repositories by creating a subdirectory, supplying a driver script and one or more execution scripts, specifying steps to obtain the raw data and, if needed, passing the resulting files downstream for additional processing. These process the raw file types provided from the repository into desired standardized formats and can access shared ARepA resources to perform common tasks such as gene ID standardization.

A typical submodule workflow includes: (1) Download the raw file of a dataset from a repository and store in the tmp directory. (2) Parse procedural configuration information specified in the etc folder and determine computational steps necessary to produce the output. (3) Execute relevant scripts in the src in the combination and order as specified by procedural configuration. (4) If override configuration exists, replace default files with user-provided files. Programmatic wrappers to these system calls are available internally for facilitation of use. For a thorough walkthrough of this feature, consult the documentation provided with the system.

\section{RESULTS}

Motivated by the need to reproducibly access and unify diverse data resources, we developed a novel approach for the retrieval, normalization, and meta-analysis of high-throughput biological data. In this study, we applied our system, ARepA (Automated Repository Acquisition), to three integrative "big data" analyses: an integration of gene networks in human prostate cancer to identify interactors with the NfкB pathway, a differential expression meta-analysis of genes and pathways up- or downregulated in the germ-free murine colon, and an integrative analysis of heterogeneous data from three model microbes to identify differentially conserved peptide secretion system pathways.

ARepA is implemented as a publicly available software package, currently providing reproducible and automated data and metadata processing for the Gene Expression Omnibus (GEO) (Barrett et al. 2011), IntAct (Kerrien et al. 2012), BioGRID (Stark et al. 2011), RegulonDB (Gama-Castro et al. 2011), STRING (Szklarczyk et al. 2011), Bacteriome (Su et al. 2008), and MPIDB (Goll et al. 2008). Investigators can retrieve normalized data and metadata in standardized formats from these repositories 
373 for any organism of interest. For six model organisms studied here in detail (human, mouse, yeast, $E$. 374 coli, B. subtilis, and $P$. aeruginosa), it currently yields data comprising more than $630 \mathrm{~K}$ interactions: $375250 \mathrm{~K}$ physical interactions (Bacteriome, BioGRID, IntAct, and MPIDB), and 380K functional 376 associations (STRING), in addition to approximately $15 \mathrm{~K}$ expression data sets covering more than 377 530K samples (GEO) with standardized formats and gene identifiers. Metadata and network data are 378 also automatically produced for each dataset and can be summarized in a portable R data file (Figure 379 1).

380

381

382

383

384

385

386

387

388

389

390

391

392

393

394

395

396

397

398

399

400

401

402

403

404

405

406

407

408

409

\section{MEN1 and ACBD6 are associated with the NFאB signaling pathway in human prostate cancer}

The NFkB gene family of transcription factors plays an important role in immune response (Hayden et al. 2006) and can promote the growth and survival of solid and haematological malignancies, including prostate cancer (Davis et al. 1999; Perkins 2012). In order to investigate this key gene family in human prostate cancer, we used ten prostate cancer gene expression datasets (Supplementary Table 1) to construct a single prostate cancer-specific functional network (see Methods); the entire process of querying, downloading, normalizing, standardizing and integrating these datasets took $<1$ hour total running time (on a $2.9 \mathrm{GHz}$ Intel Core i7 16GB machine). To identify genes highly related to NFKB gene family in human prostate cancer, we extracted a high-confidence subgraph from this integrated network around twelve NFkB signaling genes in BioCarta (see Methods, Supplementary Table 4). This query process retrieved seven additional genes known from prior work to be involved in NFkB signaling (Davis et al. 1999; Perkins 2012) (Figure 2): Tumor protein p53 (TP53), TNF receptorassociated factor 2 (TRAF2), receptor (TNFRSF)-interacting serine-threonine kinase 1 (RIPK1), mitogen-activated protein kinases (MAP3K14 and MAP2K1), and interleukins (IL1B and IL10).

Two newly-associated genes with potential relationships to NFkB and prostate cancer were also recovered: Menin (MEN1) and acyl-CoA binding domain containing 6 (ACBD6). MEN1 is a putative tumor suppressor known to physically interact with $\mathrm{NF \kappa B}$ proteins (Heppner et al. 2001). Here, we observed that in prostate cancer, MEN1 was significantly co-expressed with TP53 and IкB kinase

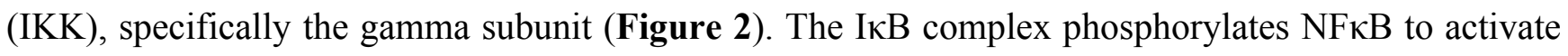
the cellular response to inflammation (Bouwmeester et al. 2004; Hacker \& Karin 2006; Wen et al. 2000), suggesting that MEN1 may represent a novel upstream regulator of the pathway in prostate cancer.

ACBD6 is a poorly-characterized protein that has been implicated in few specific areas; one of these is growth of Chlamydia trachomatis, for which it is essential in cell culture (Soupene et al. 2012). C. trachomatis has been implicated as a microbial driver in prostate carcinogenesis and has also been shown to elicit chemokine production from immune cells and non-immune cells isolated from the prostate (Oberti et al. 2011). Our prediction suggests a more specific role for ACBD6 in prostate cancer as a potential suppressor of NFאB activity and downstream inflammation, as it was highly co-

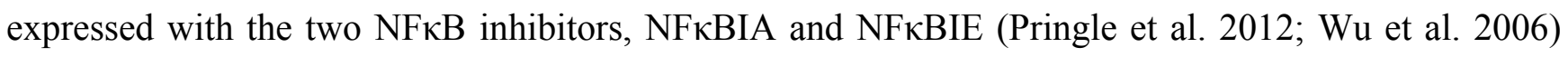
(Figure 2). This hypothesis is consistent with ACBD6's previously-reported role in bacterial growth if 
410 it represents one mechanism by which the antimicrobial response is repressed through suppression of 411 NFKB.

412 Ppar- $\alpha$ and $\operatorname{Rxr}-\alpha$ are consistently differentially expressed in a germ-free mouse intestinal meta413 analysis

414 We next used ARepA to conduct a differential expression meta-analysis of genes up- or down415 regulated in the germ-free murine gut (Supplementary Figure 1). ARepA metadata from GEO 416 included six case-control gene expression datasets containing intestinal tissue and comparing germ-free 417 and wild type mice (Supplementary Table 2). The process of identifying these datasets from 418 throughout GEO, obtaining them, and normalizing their values, gene identifiers, and metadata took $<1$ 419 hour total running time (on a $2.9 \mathrm{GHz}$ Intel Core i7 16GB machine). ARepA made the retrieved 420 datasets available as $\mathrm{R}$ data files, which were then meta-analyzed (see Methods). This identified 3,600 421 genes differentially expressed in the germ-free gut (Supplementary Table S5); these were then tested for gene set enrichment in KEGG and BioCarta pathways (Subramanian et al. 2005). Under germ-free conditions, two pathways were significantly enriched for upregulated genes, and 15 pathways were enriched for downregulated genes (Supplementary Table 3).

425

426

427

428

429

430

431

432

433

434

435

436

437

438

439

440

441

442

443

444

445

446

447

Almost all downregulated pathways under germ-free conditions were immune-related, including cytotoxic $\mathrm{T}$ lymphocyte-mediated immune response, $\mathrm{T}$ cytotoxic and $\mathrm{T}$ helper cell surface molecules, and B cell and T cell receptor signaling (Supplementary Table 3). Germ-free mice physiologically exhibit profound immune system deficiencies, including smaller lymph nodes, Peyer's patches, reduced immunoglobin production (J. Rahija 2007), reduced numbers of CD4+ T cells (Mazmanian et al. 2005) (particularly in the intestinal lamina propria (Round \& Mazmanian 2009)) and reduced numbers of intraepithelial CD8+ T cells (Round \& Mazmanian 2009). This severe dysregulation of immunity under germ-free conditions is thus consistent with existing literature and correctly detected by ARepA's automated meta-analysis.

The two upregulated pathways involved peroxisome proliferator-activated receptors (Ppar), which are nuclear hormone receptors that are activated by fatty acids and their derivatives. For example, in germfree mice, the Ppar signaling pathway from KEGG (mmu03320) was enriched for 11 significant differentially expressed genes across all datasets (Figure 3A), eight of which were Ppar- $\alpha$ target genes in lipid metabolism (Rakhshandehroo et al. 2010). Ppar- $\alpha$ is a major transcriptional regulator of lipid metabolism and is expressed in the liver and the intestinal tract. Although levels of bacteriallygenerated short-chain fatty acids are markedly decreased in the germ-free mouse intestine, Ppar- $\alpha$ is upregulated (Aoyama et al. 1998; Bookout et al. 2006; Hoverstad et al. 1985; Hussain 2013; Mukherji et al. 2013), as confirmed in our meta-analysis (Figure 3B). The upregulation of Ppar- $\alpha$ promotes the uptake, utilization, and catabolism of fatty acids by upregulating genes involved in lipid metabolism (Aoyama et al. 1998). Accordingly, downstream target genes, such as Acsl1 and Acsl5 (fatty acid binding and activation), Apoal (fatty acid uptake and metabolism), Acadm (fatty acid degradation), Fads2 (lipogenesis), or Nr1h3 (cholesterol transport and metabolism) were all consistently upregulated in expression in our meta-analysis (Figure 3A), suggesting their downstream activation in lipid 
metabolism not only in liver and skeletal muscle (Dreyer et al. 1992; Rakhshandehroo et al. 2010), but also in the intestinal tract. In addition, our meta-analysis revealed consistent upregulation of Rxr- $\alpha$ (Figure 3C) $\square \square$, a nuclear receptor that heterodimerizes with Ppar- $\alpha \square$ (Rakhshandehroo et al. 2010) and mediates the biological effects of retinoids, which play an important role in immune homeostasis in the intestine. As Ppar- $\alpha$ and Rxr- $\alpha$ synergistically regulate the target genes of Ppar- $\alpha$ within the Ppar signaling pathway from KEGG (mmu03320), we suggest that this synergistic upregulation of both genes in the intestine drives the downstream expression of fatty acid transport, lipid binding, lipid activation, and lipid degradation in the intestinal tract. This finding suggests lipid metabolism in addition to the immune system as a highly dysregulated systems in germ-free mice, potentially reflecting the altered metabolic challenges/relative energy deprivation the germ-free mouse confronts, as mice rely on their gut microbiota for optimal energy extraction from the diet.

\section{Conserved and non-conserved secretion pathways in evolutionarily diverged bacteria}

Although their genomes are relatively simple, bacteria represent billions of years of evolution; coupled with their unique mechanisms for genome plasticity, they represent an excellent system for comparative functional genomics. One example is the diversity of peptide secretion systems that modulate the interactions of bacteria with their environments. Gram-positive bacteria such as Bacillis subtilis use the general secretory (sec) pathway to translocate unfolded proteins (Ling Lin et al. 2007), and the twin arginine translocation (tat) pathway to translocate folded proteins. Gram-negative bacteria, such as E.coli, employ additional secretion systems to move proteins across their outer membranes. For example, type II secretion relies on the sec or tat pathways for translocation of proteins across the inner membrane to the periplasmic space, where they may be further modified before translocation across the outer membrane (Sandkvist 2001). These secretion strategies may be extended by Gram-negative bacteria to engage host cells. Type III secretion systems, which are not sec-dependent, allow bacteria to inject effectors, including toxins, directly into eukaryotic host cells rather than secreting them into the extracellular milieu (Coburn et al. 2007). Type VI secretion systems allow direct delivery of target proteins to prokaryotic or eukaryotic cells, but they are only sometimes associated with pathogenicity and are structurally quite distinct (Kulp \& Kuehn 2010; Silverman et al. 2012; Tseng et al. 2009). In this case study, we assess functional differences in these diverse peptide secretion systems based on a cross-species pathway comparison in B. subtilis, which is Gram-positive, and two Gram-negative bacteria: a non-pathogenic strain of E. coli, and P. aeruginosa.

Gene expression and gene interaction data were readily available for all three of these bacteria. ARepA provided an easy and reproducible way to integrate these data types (Table 2A, Table 2B). These large datasets were integrated into three species-specific functional networks using a shared gene identifier (UniRef90), which we used to investigate genes highly associated with secretion by extracting highconfidence subgraphs around the sec and tat genes and the type II, type III, and type VI secretion systems (see Methods, Supplementary Table 4). Because Gram-positive bacteria such as B. subtilis use only the general secretory ( $\mathrm{sec}$ ) and tat pathways, we extracted a high-confidence subgraph from the $B$. subtilis-specific functional network around two tat genes and nine sec genes, resulting in a highconfidence subnetwork containing 16 genes in total (Figure 4A). In contrast, the Gram-negative E. coli 
487 employs an additional secretion system (type II secretion system), and we extracted a high-confidence 488 subgraph from the $E$. coli-specific functional network around four tat genes, twelve sec genes, one type 489 I gene, and twelve type II genes (see Supplementary Table 4), resulting in a high-confidence 490 subnetwork containing 34 genes in total (Figure 4B). P. aeruginosa, as a Gram-negative with 491 pathogenic potential, additionally utilizes type I, III, V, and type VI secretion systems, and we 492 extracted a high-confidence subgraph from the $P$. aeruginosa-specific functional network around three 493 tat genes, nine sec genes, two type I genes, eleven type II genes, twelve type III genes, one type V 494 genes, and six type VI genes (see Supplementary Table 4), resulting in a high-confidence subnetwork 495 containing 49 genes in total (Figure 4C).

496 These resulting integrated molecular networks easily recover the highly functionally related gene 497 clusters within each individual system, in addition to interconnecting the systems themselves through a 498 combination of conservation and co-function. (Figure 4). As the sec and tat pathways are highly 499

500

501

502

503

504

505

506

507

508

509

510

511

512

513

514

515

516

517

518

519

520

521

522

523

524 conserved, we observed their corresponding clusters in all these bacteria to be highly functionally connected. The type II secretion pathway, which depends on the sec pathway in Gram-negative bacteria, is functionally connected to it both the $E$. coli and $P$. aeruginosa networks. Likewise, the secdependent type V pathway (Bleves et al. 2010) is also tightly connected to the sec/tat cluster in the Pseudomonas network. In contrast, the Pseudomonas type VI secretion pathway, which is tat and secindependent (Filloux et al. 2008), was much less tightly connected to the tat/sec cluster than was type II secretion. Type I secretion, which is sec-independent (Bleves et al. 2010), was as expected only weakly connected to tat/sec. The type III secretion pathway, which is also sec/tat independent (Kostakioti et al. 2005), is also unconnected to sec/tat in the Pseudomonas network, but it is tightly connected to genes from the type II secretion pathway instead. Our underlying data for integrating the Pseudomonas network show high, confident co-expression (normalized z-score $>2.0$ ) between some genes from these clusters (e.g. yscT (type III) and gspC (type II) (Kang et al. 2008; Rao et al. 2008), yscT (type III) and gspD (type II) (Williamson et al. 2012) (Figure 4)), suggesting a functional relationship between the type II and type III secretion systems in P. aeruginosa.

\section{Standardized and reproducible high-throughput data retrieval and integration}

As evidenced by these studies, ARepA provides an analysis platform that is modular and hierarchical. The system includes access to multiple repositories; within each, hierarchical data retrieval first queries all experiments from a particular source, then all datasets within each experiment, and finally all metadata for each dataset, in a manner similar to the design of the ISA standard (Rocca-Serra et al. 2010). Data from each repository can be retrieved independently if desired, as can data from individual datasets, and a user can customize multi-layered data processing pipelines in a consistent, templated manner if desired. Each repository retrieves multiple experiments (typically organized by taxon ID and publication ID), and each experiment can retrieve multiple platforms or datasets. Information is shared vertically, and data passed from parent to child analyses can include taxonomic information, desired dataset types, and repository-specific configuration information, such as type of normalization functions and machine learning algorithms. An ARepA user can generally request that all modules 
525 beneath a particular repository be executed, or that only a single submodule (e.g. one dataset) is 526 processed.

527 As an example of dedicated module implementation, the GEO module first downloads raw gene 528 expression data (SOFT or SeriesMatrix files) as well as platform annotations from the Gene Expression 529 Omnibus (Figure 5). The number of platforms used by each dataset is determined, and data for each 530 platform is built recursively. The expression data is parsed, normalized and imputed (Huttenhower et 531 al. 2008), and gene IDs are standardized using GeneMapper. Finally, metadata is created for each 532 dataset, combining automatically-derived information from the SOFT files with manually curated 533 tables if available. The final outputs are a standardized and consistent gene expression matrix encoded as tab-delimited text, corresponding metadata, a normalized co-expression network, and an $\mathrm{R}$ data file

535

536

537

538

539

540

541

542

543

544

545

546

547

548

549

550

551

552

553

554

555

556

557

558

559

560

561 containing an expression set. Other dedicated modules, such as those for interaction data, operate similarly by first downloading and parsing the requested data and directly generating tab-delimited text networks for each dataset (typically separated by taxon ID and publication ID). Standardization of interaction data and metadata are performed as described for the GEO module.

Finally, data retrieved in this environment can be integrated or meta-analyzed either heterogeneously (e.g. our bacterial study) or homogeneously (e.g. the human prostate cancer network integration and murine gut differential expression studies) by procedures such as fixed or random effects meta-analysis or network integration. The former can be directly applied to identify which genes are consistently upor down-regulated in a given organism under selected experimental conditions. This environment facilitates the identification of relevant experiments, collection of associated datasets, and application of standardized R/Bioconductor meta-analysis environments such as metaphor (Viechtbauer 2010) to gene expression data from various laboratories and platforms. Network integration studies, in contrast, can be performed for data from any source of interest (e.g. co-expression, protein-protein interactions, regulatory interactions, posttranslational modifications, etc.) by, effectively, meta-analyzing gene interactions (edges) rather genes (nodes). The simplest approach averages across normalized interaction scores (as illustrated in the human prostate cancer network integration and bacterial study) and can be extended using more sophisticated probabilistic (Franceschini et al. 2013; Lee et al. 2004; Myers \& Troyanskaya 2007; Zuberi et al. 2013) or discriminative (Chikina et al. 2009; Guan et al. 2012) models. Both gene and interaction level integrations can be performed from within ARepA directly from the standardized metadata and formatted data.

\section{ARepA data model and availability}

ARepA includes default outputs for a variety of model organisms (Table 1, Table 2) in several standardized formats, including tab-delimited expression matrices, gene interaction networks, and $\mathrm{R}$ data files (see Methods). For three eukaryotic models (human, mouse, and yeast), we have chosen UniRef100 as a standardized gene identifier; this UniRef model combines identical sequences into a single family, while for bacteria the system provides UniRef90 which are built by clustering 
562

563

564

565

566

567

568

569

570

571

572

573

574

575

576

577

578

579

580

581

582

583

584

585

586

587

588

589

590

591

592

593

594

595

596

597

598

UniRef100 sequences at 90\% sequence identity (Suzek et al. 2007). As expected, the largest amount of gene expression data is currently retrieved for human ( $>10 \mathrm{~K}$ datasets comprising $\sim 500 \mathrm{~K}$ samples) and for mouse ( $\sim \mathrm{K}$ datasets comprising $>50 \mathrm{~K}$ samples), while among the prokaryotes, most gene expression data was retrieved for E. coli (184 datasets comprising $>2 \mathrm{~K}$ samples). In terms of gene interaction data, ARepA by default produces the most interaction data for human ( $>320 \mathrm{~K}$ interactions), yeast $(>150 \mathrm{~K}$ interactions), and $E$. coli $(>100 \mathrm{~K}$ interactions). Functional associations across all six organisms tended to comprise more interactions $(>380 \mathrm{~K})$ than did physical protein-protein interaction data alone $(>250 \mathrm{~K})$. In terms of physical interactions, BioGRID yielded twice as many physical interactions as IntAct across the eukaryotes, while most of the physical interactions in the three prokaryotes were retrieved from IntAct. Since Bacteriome and RegulonDB are databases for physical and regulatory interactions specific to $E$. coli, their contribution to physical and regulatory interactions across species was limited, and the physical interactions derived from Bacteriome make up more than $50 \%$ of all physical interactions in E. coli.

\section{DISCUSSION}

ARepA was developed to provide an automatic, consistent, reproducible, and high-throughput method of accessing, retrieving, standardizing, processing, and integrating diverse, heterogeneous data resources, suitable for large integrative analyses and meta-analyses in translational and bioinformatic research studies. To illustrate ARepA's utility in such studies, we have described here three biological studies demonstrating the utility of ARepA for integrative analyses. When focusing on human data, ARepA's metadata database allowed us to identify and standardize ten human prostate cancer gene expression datasets from GEO, which were subsequently meta-analyzed, while a subsequent coexpression network analysis recovered new candidate genes with potential roles in the NFkB signaling pathway in prostate cancer. A similar example in mouse integrated six gene expression datasets selected by querying ARepA for metadata indicating germ-free and intestinal tissue conditions. Finally, multiple data types from three model microbes were integrated to assess functional differences in peptide secretion systems.

In this study, we correctly recovered and extended the molecular network of the NFאB signaling pathway in human prostate cancer by using this environment to identify ten tissue-specific human expression datasets and subsequently meta-analyze them across six different array platforms. The total computing time needed to identify, normalize, and integrate these datasets was approximately 1 hour. In contrast, performing a comparable process using Taverna (Hull et al. 2006) required 8 total main steps: 1) downloading gene expression data from GEO, 2) loading and accessing gene expression data (maxd software: maxdLoad2 and maxdBrowse (Hancock et al. 2005)), 3) attaching (curated) metadata $(\operatorname{maxdLoad} 2), 4)$ normalizing and pre-processing the raw data (Taverna/Biomoby/runPreprocessing and AffyArrayNormalization (Kawas et al. 2006) or RShell (Wassink et al. 2009)), 5) identifying and 
599 downloading dataset specific mapping files, 6) mapping from probeset to gene identifier 600 (Taverna/Biomoby/GeneExpressionAnnotation, BeanShell scripting language), 7) generating co601 expression networks (Huttenhower et al. 2008), and 8) building an integrative expression set object. 602 Running a similar process using GEOquery (Davis \& Meltzer 2007) required seven total main steps: 1) 603 downloading expression data from GEO (R/GEOquery/getGEO), 2) normalizing and pre-processing 604 the raw data (R/affy/RMA (Gautier et al. 2004; Irizarry et al. 2003)), 3) identifying and downloading 605 dataset specific mapping files, 4) mapping from probeset to gene identifier for non-standard microarray 606 platforms, 5) attaching (curated) metadata, 6) generating co-expression networks (Huttenhower et al. 607 2008), and 7) building an integrated expression set object. Using ARepA requires none of these steps, 608 as it is specifically constructed for acquisition, standardization, query, and integration tasks, resulting 609 in co-expression/interaction networks and expression sets without the need for additional intervention.

610 Performing this task in ARepA allowed us to recover several genes already associated with NFKB and 611 prostate cancer (Davis et al. 1999; Perkins 2012), in addition to original findings including MEN1 as a 612 novel putative upstream regulator (Bouwmeester et al. 2004; Hacker \& Karin 2006; Heppner et al. 613 2001; Wen et al. 2000) and ACBD6 as a potential downstream suppressor of NFאB-activation (Oberti 614 et al. 2011; Pringle et al. 2012; Soupene et al. 2012; Wu et al. 2006). MEN1 was linked to cancer, 615 616 prostate cancer and NFאB by two main data sources. First, MEN1 co- immunoprecipitated with the $\mathrm{NF} \kappa \mathrm{B}$ proteins $\mathrm{p} 50$, p52, and $\mathrm{p} 65$ in vitro and in vivo, revealing MEN1 as a repressor of NFkB/p65mediated transcriptional activation and a tumor suppressor protein (Heppner et al. 2001). Second, MEN1 and NFאB/p65 were co-expressed in hepatocellular carcinoma (HCC) tissues, adjacent normal liver tissues and various cell lines (including THLE-3, HepG2, Hep3B, HuH-7 and Li-7 cells)(Gang et al. 2013). Although no mechanism was previously proposed, heterozygous Men1 mutant mice $\left(\right.$ Men $1^{+/-}$ ) carrying an inactivated Menl allele (Bertolino et al. 2003) and mixed with C57BL6/129-Sv mouse strains developed prostate cancer as compared with wild-type $\left(M e n 1^{+/+}\right)$littermates in aged mice (18 to 26 months) (Seigne et al. 2010). The strong functional linkage of MEN1 with IKBkG in our data, as well as p53, supports the previously suggested role for this gene product upstream of the NFkB pathway as a tumor suppressor by inhibition of NFkB-mediated transactivation. This could be further validated by, for example, siRNA knockdown of MEN1 in LAPC4 cells to determine whether MEN1 silencing results in more rapid cellular growth in a hormone-depleted state.

628

629

630

631

632

633

634

635

636

637

To the best of our knowledge, ACBD6 has not been directly linked to cancer, although its expression promotes C. trachomatis-mediated carcinogenesis in vitro (Soupene et al. 2012). A homologous protein also carrying the acyl-CoA binding domain, ACBD3, was highly expressed in primary pigmented nodular adrenocortical disease (PPNAD) tissues (Liu et al. 2003), lung cancer tissue in treated patients that did not respond to gefitinib (Kakiuchi et al. 2004), colonic mucosa cancer tissue upon pretreatment versus post-treatment with celecoxib (Glebov et al. 2006), and bone tissue in osteosarcoma patients (Jones et al. 2012), supporting a role as a potential oncogene (Fan et al. 2010). Additionally, another homologous protein, ACBD4, was highly up-regulated in cell lines from different tumor types (including MCF-7 breast adenocarcinoma, HeLa cervical cancer, HT1080 sarcoma, KB laryngeal carcinoma, SW480 colon carcinoma, Hep-2 oral carcinoma and D54 glioma) 
638 after inducing by the histone deacetylase inhibitor valproic acid (VPA) (Chavez-Blanco et al. 2006), 639 while Acyl-CoA synthetase (ACS), which converts long-chain fatty acids into acyl-CoA, is 640 overexpressed in different cancer cell lines (including glioma SF268 and U251 cells) (Mashima et al. 641 2009), lung cancer (Pei et al. 2013), and glioblastoma (Pei et al. 2009). Based on such links between 642 acyl-CoA binding domains, their synthetase, and cancer, as well as our data that predicted a strong

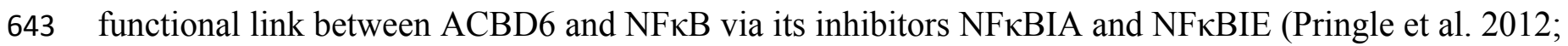
644 Wu et al. 2006) (Figure 2), we suggest a potential role of ACBD6 in prostate cancer as an upstream 645 suppressor protein of NFkB activation. This could be experimentally validated by screening series of 646 647 648

649 650

651

652

653

654

655

656

657

658

659

660

661

662

663

664

665

666

667 668 669 670 671

672

673

674

675 prostate cancer cells (LAPC4, LNCaP) to determine whether ACBD6 is present, and in those cell lines in which it is present, siRNA knockdown of ACBD6 could be performed do to functional assays, such as proliferation in hormone sensitive (LNCaP) and hormone resistant cell lines (LNCaP-abl).

Our study of host-microbiota interactions focused on identifying transcripts differentially expressed in the germ-free mouse colon. As expected based on prior knowledge of germ-free mice, there was strong differential expression of immune and carbohydrate metabolism-related genes. Meta-analysis showed that genes from the Ppar signaling pathway were highly differentially expressed under germ-free conditions. Specifically, Ppar- $\alpha$, a major regulator of lipid metabolism was upregulated. Ppar signaling overall plays key roles in regulating energy metabolism, cellular proliferation and differentiation in many tissues, including the intestine, kidney, heart, and lung (Braissant et al. 1996; Nephew et al. 1996). Dysregulated Ppar signaling has been implicated in metabolic syndrome, for which there is an increasing appreciation for the role of gut microbiota (Aitken \& Gewirtz 2013; Tremaroli \& Backhed 2012). Prior work using Ppar- $\alpha \square$ deficient mice coupled with gnotobiotic re-derivation suggests that Ppar- $\alpha$ does not function in the microbiota-dependent regulation of fat storage (Backhed et al. 2004). Thus to determine its specific role in this predicted context, follow-up work could include knockout mice for these genes (inclusive of conditional mice-deficiencies within intestinal epithelial or immune cells within the gut), with or without defined microbial consortia, screened using different diets (e.g. high fat, methionine and choline deficient). The observation that the germ-free state resulted in significant perturbations to Ppar signaling pathways suggests that how microbial consortia and their metabolites affect host regulation of obesity-related inflammation, insulin responsiveness and cholesterol homeostasis should be further investigated.

In the third study, multiple data types from three model microbes (B. subtilis, E. coli, and $P$. aeruginosa) were integrated to correctly identify differentially conserved peptide secretion system pathways in the Gram-positive bacterium B. subtilis and the two Gram-negative bacteria E. coli and $P$. aeruginosa. The sec and tat pathways are highly conserved across all three bacteria and were easily retrieved during this analysis, as were type II secretion in E. coli and P. aeruginosa and the type I, III, and V, and VI pathways in P. aeruginosa (Kostakioti et al. 2005). The E. coli network was built from expression data from strain K12 (taxonomy ID 83333). While some E. coli strains (e.g. (e.g. strain O157:H7) have type III secretion systems (Sharma et al. 2012), strain K12 does not. Although it has a (type I) TolC gene, it also lacks a complete HlyABCD type I secretion operon (Bakkes et al. 2010) We 
676

677

678

679

680

681

682

683

684

685

686

687

688

689

690

691

692

693

694

695

696

697

698

699

700

701

702

703

704

705

706

707

708

709

710

711

712

713

714

populated our K12 expression-based network with only the secretion systems possessed by strain K12, and correctly recovered strong sec/tat co-expression, and strong sec/tat/type II co-expression.

In $P$. aeruginosa, there was a strong type II/III secretion link as well as strong tat/sec coordination, while type VI secretion was only weakly connected to sec/tat. Type V secretion, which is secdependent (Bleves et al. 2010), was strongly linked to sec/tat. The strong coregulation of type II and III secretion in Pseudomonas is potentially due to their joint role in pathogenesis; for example, in Pseudomonas-mediated pneumonia, type II secretion is used to secrete proteases such as LasA and LasB, phospholipase $\mathrm{H}$, and lipolytic enzymes which degrade host tissue; type II secretion allows Pseudomonas infections to persist in mice (Jyot et al. 2011). The toxins secreted by the type III secretion system are complimentary, but focused on immune inhibition rather than tissue destruction. For example, ExoT slows wound healing and inhibits bacterial internalization by macrophages, and ExoS induces lymphocyte apoptosis (Schulert et al. 2003). Pseudomonas that lacks type III secretion is much more vulnerable to immediate clearance by neutrophils (Sun et al. 2012). Thus, the type II and type III secretion systems in Pseudomonas work together to help it persist in the host in infection. Type VI secretion in Pseudomonas is much less well-characterized, but proteins secreted by this mechanism do not contain sec or tat signals (Pallen et al. 2003). While type VI secretion has been implicated in biofilm production (Zhang et al. 2011), the operon associated with biofilm is repressed by quorum sensing (Bleves et al. 2010), so an association with sec and tat that is driven by quorum sensing for the purpose of biofilm production is unlikely.

In comparison with existing bioinformatics workflow tools like Taverna (Hull et al. 2006) and Galaxy (Giardine et al. 2005; Goecks et al. 2010), ARepA is specifically tuned to download, normalize, and meta-analyze very large and diverse collections of high-throughput biological data. While users can author new modules for other analyses of the resulting integrated data, the system is tuned to facilitate this task seamlessly out of the box. Galaxy, on the other hand, focuses primarily on real-time, interactive analyses, typically of a few datasets of interest through a graphical user interface. This makes it very flexible when developing new or customized analysis workflows of particular datasets, but less easily adaptable to bulk processing of especially diverse data. Taverna, alternatively, provides an extensive platform for combining bioinformatics tools in a graphical environment, but again it does not directly facilitate bulk data download, normalization, and integration. By uniformly normalizing and providing metadata for results deposited across many high-throughput data repositories, ARepA makes it easy to integrate all relevant data types for organisms, phenotypes, diseases, or molecular targets of interest.

One of the most important application areas for an environment such as ARepA is in translational meta-analyses, which relies on large data collections from multiple sources to ensure cross-study validation (Ganzfried et al. 2013; Griffith et al. 2006). ARepA supports such efforts in two ways. First, it effectively integrates data of heterogeneous format and origin without the need for manual curation or user intervention. This is true both for data normalization and for metadata collection, the latter of which is also integrated into a single, searchable database from across multiple repositories. ARepA's 
715

716

717

718

719

720

721

722

723

724

725

726

727

728

729

730

731

732

733

734

735

736

737

738

739

740

741

742

743

744

745

746

747

748

749

750

751

752

753

automated processing can thus be used as a first step in particularly highly targeted manual curation if desired, as has been the case in our own previous studies (Ganzfried et al. 2013; Waldron et al. 2012). Second, ARepA provides complete provenance and reproducibility for all data retrieved, as this is similarly a priority in pre-clinical research. The data processing and normalization steps carried out by ARepA can be modified or added to by a user if desired, ensuring that exactly the same steps are carried out at each execution for each dataset. This remains practical even when handling large data collections since ARepA's dependency-tracking architecture ensures execution only when the input data source has changed or new analyses are added.

A second potentially important environment for ARepA is as a basis for "in-house" repositories for groups supporting internal data or focusing on specific types (e.g. stem cell data). This provides a convenient way to start from public repositories by first screening metadata for areas of interest. This screen can be added to ARepA's data retrieval process for each source repository, ensuring that only data of interest is downloaded and updated each time; the latter is important since public repositories do updated regularly and on a varying basis (e.g. GEO is updated continuously, BioGRID once a month, and STRING once to twice per year). Privately maintained data can be appended by adding a new module to ARepA that fetches data locally, allowing it to be standardized to the same format as that of the other repositories for integration. ARepA is generally designed to be customizable to individual user environments, allowing modification of data retrieval or processing scripts while still taking advantage of its dependency-tracking system. Public data are typically fetched via ftp or http; however, a user can easily acquire data using other protocols such as ssh, Aspera, or simple local copies or links. This flexibility in design allows users to integrate publically available data with privately maintained data to conduct in-silico experiments within a single consistent environment.

Since any high-throughput genomic assays are susceptible to batch effects (Leek et al. 2010), analysts performing meta-analyses should be aware of their potential impact. Batch correction methods in general either require a priori knowledge of the batches (e.g. for empirical Bayes adjustment(Johnson et al. 2007)) or are specific to the outcome variable of a particular differential expression (Leek et al. 2012), as has been previously reviewed (Chen et al. 2011; Lazar et al. 2013). Thus batch correction is a study-specific issue that cannot be resolved solely by a high-throughput tool such as ARepA, although it provides a framework within which individual datasets can be easily handled. Although studyspecific sensitivity analysis of batch correction is warranted when possible, several studies have found that traditional meta-analysis approaches are robust to batch effects and that explicit batch correction may be unhelpful for independent validation of differential expression (Re et al. 2014) or prediction models (Riester et al. 2014; Waldron et al. 2014).

Like any computational environment, this is one of a number of routes available for future expansions to ARepA. An obvious next step is the inclusion of additional repository modules, with large and wellannotated sources such as ArrayExpress (Rustici et al. 2013), Refseq (Pruitt et al. 2005), ENCODE(Consortium 2012), or RNA-sequencing data representing excellent candidates. This will likewise allow the integration of additional data types such as genomic sequences, regulatory elements, and high quality expression data that would broaden the set of data to be considered for subsequent 
754

755

756

757

758

759

760

761

762

763

764

765

766

767

768

769

770

771

772

773

774

775

776

777

778

779

780

781

782

783

784

785

786 meta-analyses. Additionally, while the provenance of integrated data is currently tracked by recording each processing step, ARepA does not currently "version control" the analysis software it uses (e.g. R packages or external binaries), which is a possible addition for future versions. The additional of a graphical user interface will make ARepA more accessible for a broader user community and increase its usability, as will a unified configuration interface (e.g. a single, XML-based configuration file) to more easily change parameters. The current version of ARepA thus represents only the first step in the ongoing development of an environment for reproducible "big data" research, which we hope will be both expandable by bioinformaticians and useful for targeted biological studies.

\section{ACKNOWLEDGEMENTS}

We thank Timothy Tickle, Svitlana Tyekucheva, Markus Riester, Arthur Brady, Owen White, and the members of the Huttenhower lab for their input and thoughtful discussions on testing and using ARepA.

\section{FUNDING}

This work was supported by DoD W81XWH-11-1-0379 to CS, by Danone Research grant PLF-5972GD to WSG, and by NSF CAREER DBI-1053486 and NIH R01HG005969 and U54DE023798 to CH.

Conflict of interest: The authors have no conflict of interest.

\section{REFERENCES}

Affymetrix. 2002. Statistical algorithms description document. Santa Clara, CA: Affymetrix Inc.

Aitken JD, and Gewirtz AT. 2013. Gut microbiota in 2012: Toward understanding and manipulating the gut microbiota. Nat Rev Gastroenterol Hepatol 10:72-74.

Aoyama T, Peters JM, Iritani N, Nakajima T, Furihata K, Hashimoto T, and Gonzalez FJ. 1998. Altered constitutive expression of fatty acid-metabolizing enzymes in mice lacking the peroxisome proliferatoractivated receptor alpha (PPARalpha). J Biol Chem 273:5678-5684.

Backhed F, Ding H, Wang T, Hooper LV, Koh GY, Nagy A, Semenkovich CF, and Gordon JI. 2004. The gut microbiota as an environmental factor that regulates fat storage. Proc Natl Acad Sci U S A 101:1571815723. 
Baggerly KA, and Coombes KR. 2009. Deriving chemosensitivity from cell lines: Forensic bioinformatics and reproducible research in high-throughput biology. The Annals of Applied Statistics 3:1309-1334.

Bakkes PJ, Jenewein S, Smits SH, Holland IB, and Schmitt L. 2010. The rate of folding dictates substrate secretion by the Escherichia coli hemolysin type 1 secretion system. J Biol Chem 285:40573-40580.

Barrett T, Troup DB, Wilhite SE, Ledoux P, Evangelista C, Kim IF, Tomashevsky M, Marshall KA, Phillippy KH, Sherman PM, Muertter RN, Holko M, Ayanbule O, Yefanov A, and Soboleva A. 2011. NCBI GEO: archive for functional genomics data sets--10 years on. Nucleic Acids Res 39:D1005-1010.

Barrett T, Wilhite SE, Ledoux P, Evangelista C, Kim IF, Tomashevsky M, Marshall KA, Phillippy KH, Sherman PM, Holko M, Yefanov A, Lee H, Zhang N, Robertson CL, Serova N, Davis S, and Soboleva A. 2013. NCBI GEO: archive for functional genomics data sets--update. Nucleic Acids Res 41:D991-995.

Barseghian D, Altintas I, Jones MB, Crawl D, Potter N, Gallagher J, Cornillon P, Schildhauer M, Borer ET, and Seabloom EW. 2010. Workflows and extensions to the Kepler scientific workflow system to support environmental sensor data access and analysis. Ecological Informatics 5:42-50.

Benjamini Y, \& Hochberg, Y. . 1995. Controlling the false discovery rate: a practical and powerful approach to multiple testing. Journal of the Royal Statistical Society Series B (Methodological):289-300.

Bertolino P, Radovanovic I, Casse H, Aguzzi A, Wang ZQ, and Zhang CX. 2003. Genetic ablation of the tumor suppressor menin causes lethality at mid-gestation with defects in multiple organs. Mech Dev 120:549560.

Bleves S, Viarre V, Salacha R, Michel GP, Filloux A, and Voulhoux R. 2010. Protein secretion systems in Pseudomonas aeruginosa: A wealth of pathogenic weapons. Int J Med Microbiol 300:534-543.

Bookout AL, Jeong Y, Downes M, Yu RT, Evans RM, and Mangelsdorf DJ. 2006. Anatomical profiling of nuclear receptor expression reveals a hierarchical transcriptional network. Cell 126:789-799.

Bouwmeester T, Bauch A, Ruffner H, Angrand PO, Bergamini G, Croughton K, Cruciat C, Eberhard D, Gagneur J, Ghidelli S, Hopf C, Huhse B, Mangano R, Michon AM, Schirle M, Schlegl J, Schwab M, Stein MA, Bauer

A, Casari G, Drewes G, Gavin AC, Jackson DB, Joberty G, Neubauer G, Rick J, Kuster B, and Superti-Furga G. 2004. A physical and functional map of the human TNF-alpha/NF-kappa B signal transduction pathway. Nat Cell Biol 6:97-105.

Braissant O, Foufelle F, Scotto C, Dauca M, and Wahli W. 1996. Differential expression of peroxisome proliferator-activated receptors (PPARs): tissue distribution of PPAR-alpha, -beta, and -gamma in the adult rat. Endocrinology 137:354-366.

Chan SK, Griffith OL, Tai IT, and Jones SJ. 2008. Meta-analysis of colorectal cancer gene expression profiling studies identifies consistently reported candidate biomarkers. Cancer Epidemiol Biomarkers Prev 17:543-552.

Charaniya S, Hu WS, and Karypis G. 2008. Mining bioprocess data: opportunities and challenges. Trends Biotechnol 26:690-699.

Chatr-Aryamontri A, Breitkreutz BJ, Heinicke S, Boucher L, Winter A, Stark C, Nixon J, Ramage L, Kolas N, O'Donnell L, Reguly T, Breitkreutz A, Sellam A, Chen D, Chang C, Rust J, Livstone M, Oughtred R, Dolinski K, and Tyers M. 2013. The BioGRID interaction database: 2013 update. Nucleic Acids Res 41:D816-823.

Chavez-Blanco A, Perez-Plasencia C, Perez-Cardenas E, Carrasco-Legleu C, Rangel-Lopez E, Segura-Pacheco B, Taja-Chayeb L, Trejo-Becerril C, Gonzalez-Fierro A, Candelaria M, Cabrera G, and Duenas-Gonzalez A. 2006. Antineoplastic effects of the DNA methylation inhibitor hydralazine and the histone deacetylase inhibitor valproic acid in cancer cell lines. Cancer Cell Int 6:2.

Chen C, Grennan K, Badner J, Zhang D, Gershon E, Jin L, and Liu C. 2011. Removing batch effects in analysis of expression microarray data: an evaluation of six batch adjustment methods. PLoS One 6:e17238.

Chikina MD, Huttenhower C, Murphy CT, and Troyanskaya OG. 2009. Global prediction of tissue-specific gene expression and context-dependent gene networks in Caenorhabditis elegans. PLoS Comput Biol 5:e1000417. 
Coburn B, Sekirov I, and Finlay BB. 2007. Type III secretion systems and disease. Clinical microbiology reviews 20:535-549.

Consortium TEP. 2012. An integrated encyclopedia of DNA elements in the human genome. Nature 489:57-74. Davis JN, Kucuk O, and Sarkar FH. 1999. Genistein inhibits NF-kappa B activation in prostate cancer cells. Nutr Cancer 35:167-174.

Davis S, and Meltzer PS. 2007. GEOquery: a bridge between the Gene Expression Omnibus (GEO) and BioConductor. Bioinformatics 23:1846-1847.

Dowell RD, Jokerst RM, Day A, Eddy SR, and Stein L. 2001. The distributed annotation system. BMC Bioinformatics 2:7.

Dressman HK, Berchuck A, Chan G, Zhai J, Bild A, Sayer R, Cragun J, Clarke J, Whitaker RS, Li L, Gray J, Marks J, Ginsburg GS, Potti A, West M, Nevins JR, and Lancaster JM. 2007. An integrated genomic-based approach to individualized treatment of patients with advanced-stage ovarian cancer. J Clin Oncol 25:517-525.

Dreyer C, Krey G, Keller H, Givel F, Helftenbein G, and Wahli W. 1992. Control of the peroxisomal betaoxidation pathway by a novel family of nuclear hormone receptors. Cell 68:879-887.

Fan J, Liu J, Culty M, and Papadopoulos V. 2010. Acyl-coenzyme A binding domain containing 3 (ACBD3; PAP7; GCP60): an emerging signaling molecule. Prog Lipid Res 49:218-234.

Filloux A, Hachani A, and Bleves S. 2008. The bacterial type VI secretion machine: yet another player for protein transport across membranes. Microbiology 154:1570-1583.

Franceschini A, Szklarczyk D, Frankild S, Kuhn M, Simonovic M, Roth A, Lin J, Minguez P, Bork P, von Mering C, and Jensen LJ. 2013. STRING v9.1: protein-protein interaction networks, with increased coverage and integration. Nucleic Acids Res 41:D808-815.

Gama-Castro S, Salgado H, Peralta-Gil M, Santos-Zavaleta A, Muniz-Rascado L, Solano-Lira H, Jimenez-Jacinto V, Weiss V, Garcia-Sotelo JS, Lopez-Fuentes A, Porron-Sotelo L, Alquicira-Hernandez S, Medina-Rivera A, Martinez-Flores I, Alquicira-Hernandez K, Martinez-Adame R, Bonavides-Martinez C, Miranda-Rios J, Huerta AM, Mendoza-Vargas A, Collado-Torres L, Taboada B, Vega-Alvarado L, Olvera M, Olvera L, Grande R, Morett E, and Collado-Vides J. 2011. RegulonDB version 7.0: transcriptional regulation of Escherichia coli K-12 integrated within genetic sensory response units (Gensor Units). Nucleic Acids Res 39:D98-105.

Gang D, Hongwei H, Hedai L, Ming Z, Qian H, and Zhijun L. 2013. The tumor suppressor protein menin inhibits NF-kappaB-mediated transactivation through recruitment of Sirt1 in hepatocellular carcinoma. Mol Biol Rep 40:2461-2466.

Ganzfried BF, Riester M, Haibe-Kains B, Risch T, Tyekucheva S, Jazic I, Wang XV, Ahmadifar M, Birrer MJ, Parmigiani G, Huttenhower C, and Waldron L. 2013. curatedOvarianData: clinically annotated data for the ovarian cancer transcriptome. Database (Oxford) 2013:bat013.

Garman KS, Acharya CR, Edelman E, Grade M, Gaedcke J, Sud S, Barry W, Diehl AM, Provenzale D, Ginsburg GS, Ghadimi BM, Ried T, Nevins JR, Mukherjee S, Hsu D, and Potti A. 2008. A genomic approach to colon cancer risk stratification yields biologic insights into therapeutic opportunities. Proc Natl Acad Sci U S A 105:19432-19437.

Gautier L, Cope L, Bolstad BM, and Irizarry RA. 2004. affy--analysis of Affymetrix GeneChip data at the probe level. Bioinformatics 20:307-315.

Gentleman R. 2005. Reproducible research: a bioinformatics case study. Stat Appl Genet Mol Biol 4:Article2. Giardine B, Riemer C, Hardison RC, Burhans R, Elnitski L, Shah P, Zhang Y, Blankenberg D, Albert I, Taylor J, Miller W, Kent WJ, and Nekrutenko A. 2005. Galaxy: a platform for interactive large-scale genome analysis. Genome Res 15:1451-1455.

Glebov OK, Rodriguez LM, Lynch P, Patterson S, Lynch H, Nakahara K, Jenkins J, Cliatt J, Humbyrd CJ, Denobile J, Soballe P, Gallinger S, Buchbinder A, Gordon G, Hawk E, and Kirsch IR. 2006. Celecoxib treatment alters 
the gene expression profile of normal colonic mucosa. Cancer Epidemiol Biomarkers Prev 15:13821391.

Goecks J, Nekrutenko A, and Taylor J. 2010. Galaxy: a comprehensive approach for supporting accessible, reproducible, and transparent computational research in the life sciences. Genome Biol 11:R86.

Goll J, Rajagopala SV, Shiau SC, Wu H, Lamb BT, and Uetz P. 2008. MPIDB: the microbial protein interaction database. Bioinformatics 24:1743-1744.

Griffith OL, Melck A, Jones SJ, and Wiseman SM. 2006. Meta-analysis and meta-review of thyroid cancer gene expression profiling studies identifies important diagnostic biomarkers. J Clin Oncol 24:5043-5051.

Guan Y, Gorenshteyn D, Burmeister M, Wong AK, Schimenti JC, Handel MA, Bult CJ, Hibbs MA, and Troyanskaya OG. 2012. Tissue-specific functional networks for prioritizing phenotype and disease genes. PLoS Comput Biol 8:e1002694.

Hacker H, and Karin M. 2006. Regulation and function of IKK and IKK-related kinases. Sci STKE 2006:re13.

Hancock D, Wilson M, Velarde G, Morrison N, Hayes A, Hulme H, Wood AJ, Nashar K, Kell DB, and Brass A. 2005. maxdLoad2 and maxdBrowse: standards-compliant tools for microarray experimental annotation, data management and dissemination. BMC Bioinformatics 6:264.

Hayden MS, West AP, and Ghosh S. 2006. NF-kappaB and the immune response. Oncogene 25:6758-6780. Heppner C, Bilimoria KY, Agarwal SK, Kester M, Whitty L, Guru SC, Chandrasekharappa SC, Collins FS, Spiegel AM, and Marx SJ. 2001. The tumor suppressor protein menin interacts with NF-kappaB proteins and inhibits NF-kappaB-mediated transactivation. Oncogene 20:4917.

Hoverstad T, Midtvedt T, and Bohmer T. 1985. Short-chain fatty acids in intestinal content of germfree mice monocontaminated with Escherichia coli or Clostridium difficile. Scand J Gastroenterol 20:373-380.

Hull D, Wolstencroft K, Stevens R, Goble C, Pocock MR, Li P, and Oinn T. 2006. Taverna: a tool for building and running workflows of services. Nucleic Acids Res 34:W729-732.

Hussain MM. 2013. Metabolism: Gut microbiota modulates diurnal secretion of glucocorticoids. Nat Rev Endocrinol 9:444-446.

Huttenhower C, Haley EM, Hibbs MA, Dumeaux V, Barrett DR, Coller HA, and Troyanskaya OG. 2009. Exploring the human genome with functional maps. Genome Res 19:1093-1106.

Huttenhower C, Schroeder M, Chikina MD, and Troyanskaya OG. 2008. The Sleipnir library for computational functional genomics. Bioinformatics 24:1559-1561.

loannidis JP, Allison DB, Ball CA, Coulibaly I, Cui X, Culhane AC, Falchi M, Furlanello C, Game L, Jurman G, Mangion J, Mehta T, Nitzberg M, Page GP, Petretto E, and van Noort V. 2009. Repeatability of published microarray gene expression analyses. Nat Genet 41:149-155.

Irizarry RA, Hobbs B, Collin F, Beazer-Barclay YD, Antonellis KJ, Scherf U, and Speed TP. 2003. Exploration, normalization, and summaries of high density oligonucleotide array probe level data. Biostatistics 4:249-264.

J. Rahija R. 2007. Chapter 7 - Gnotobiotics. In: James GF, Muriel TD, Fred WQ, Stephen WB, Christian EN, Abigail L. SmithA2 - James G. Fox MTDFWQSWBCEN, and Abigail LS, eds. The Mouse in Biomedical Research (Second Edition). Burlington: Academic Press, 217-233.

Johnson WE, Li C, and Rabinovic A. 2007. Adjusting batch effects in microarray expression data using empirical Bayes methods. Biostatistics 8:118-127.

Jones KB, Salah Z, Del Mare S, Galasso M, Gaudio E, Nuovo GJ, Lovat F, LeBlanc K, Palatini J, Randall RL, Volinia S, Stein GS, Croce CM, Lian JB, and Aqeilan RI. 2012. miRNA signatures associate with pathogenesis and progression of osteosarcoma. Cancer Res 72:1865-1877.

Jyot J, Balloy V, Jouvion G, Verma A, Touqui L, Huerre M, Chignard M, and Ramphal R. 2011. Type II secretion system of Pseudomonas aeruginosa: in vivo evidence of a significant role in death due to lung infection. J Infect Dis 203:1369-1377. 
Kakiuchi S, Daigo Y, Ishikawa N, Furukawa C, Tsunoda T, Yano S, Nakagawa K, Tsuruo T, Kohno N, Fukuoka M, Sone S, and Nakamura Y. 2004. Prediction of sensitivity of advanced non-small cell lung cancers to gefitinib (Iressa, ZD1839). Hum Mol Genet 13:3029-3043.

Kang Y, Nguyen DT, Son MS, and Hoang TT. 2008. The Pseudomonas aeruginosa PsrA responds to long-chain fatty acid signals to regulate the fadBA5 beta-oxidation operon. Microbiology 154:1584-1598.

Kapushesky M, Adamusiak T, Burdett T, Culhane A, Farne A, Filippov A, Holloway E, Klebanov A, Kryvych N, Kurbatova N, Kurnosov P, Malone J, Melnichuk O, Petryszak R, Pultsin N, Rustici G, Tikhonov A, Travillian RS, Williams E, Zorin A, Parkinson H, and Brazma A. 2012. Gene Expression Atlas update--a value-added database of microarray and sequencing-based functional genomics experiments. Nucleic Acids Res 40:D1077-1081.

Kauffmann A, Gentleman R, and Huber W. 2009. arrayQualityMetrics--a bioconductor package for quality assessment of microarray data. Bioinformatics 25:415-416.

Kawas E, Senger M, and Wilkinson MD. 2006. BioMoby extensions to the Taverna workflow management and enactment software. BMC Bioinformatics 7:523.

Kerrien S, Aranda B, Breuza L, Bridge A, Broackes-Carter F, Chen C, Duesbury M, Dumousseau M, Feuermann M, Hinz U, Jandrasits C, Jimenez RC, Khadake J, Mahadevan U, Masson P, Pedruzzi I, Pfeiffenberger E, Porras P, Raghunath A, Roechert B, Orchard S, and Hermjakob H. 2012. The IntAct molecular interaction database in 2012. Nucleic Acids Res 40:D841-846.

Kinsella RJ, Kahari A, Haider S, Zamora J, Proctor G, Spudich G, Almeida-King J, Staines D, Derwent P, Kerhornou A, Kersey P, and Flicek P. 2011. Ensembl BioMarts: a hub for data retrieval across taxonomic space. Database (Oxford) 2011:bar030.

Kostakioti M, Newman CL, Thanassi DG, and Stathopoulos C. 2005. Mechanisms of protein export across the bacterial outer membrane. Journal of bacteriology 187:4306-4314.

Kulp A, and Kuehn MJ. 2010. Biological functions and biogenesis of secreted bacterial outer membrane vesicles. Annu Rev Microbiol 64:163-184.

Lazar C, Meganck S, Taminau J, Steenhoff D, Coletta A, Molter C, Weiss-Solis DY, Duque R, Bersini H, and Nowe A. 2013. Batch effect removal methods for microarray gene expression data integration: a survey. Brief Bioinform 14:469-490.

Lee I, Date SV, Adai AT, and Marcotte EM. 2004. A probabilistic functional network of yeast genes. Science 306:1555-1558.

Leek JT, Johnson WE, Parker HS, Jaffe AE, and Storey JD. 2012. The sva package for removing batch effects and other unwanted variation in high-throughput experiments. Bioinformatics 28:882-883.

Leek JT, Scharpf RB, Bravo HC, Simcha D, Langmead B, Johnson WE, Geman D, Baggerly K, and Irizarry RA. 2010. Tackling the widespread and critical impact of batch effects in high-throughput data. Nat Rev Genet 11:733-739.

Ling Lin F, Zi Rong X, Wei Fen L, Jiang Bing S, Ping L, and Chun Xia H. 2007. Protein secretion pathways in Bacillus subtilis: implication for optimization of heterologous protein secretion. Biotechnol Adv 25:1-12. Liu J, Li H, and Papadopoulos V. 2003. PAP7, a PBR/PKA-Rlalpha-associated protein: a new element in the relay of the hormonal induction of steroidogenesis. J Steroid Biochem Mol Biol 85:275-283.

Lu J, Getz G, Miska EA, Alvarez-Saavedra E, Lamb J, Peck D, Sweet-Cordero A, Ebert BL, Mak RH, Ferrando AA, Downing JR, Jacks T, Horvitz HR, and Golub TR. 2005. MicroRNA expression profiles classify human cancers. Nature 435:834-838.

Lynch C. 2008. Big data: How do your data grow? Nature 455:28-29.

Marx V. 2013. Biology: The big challenges of big data. Nature 498:255-260.

Mashima T, Sato S, Okabe S, Miyata S, Matsuura M, Sugimoto Y, Tsuruo T, and Seimiya H. 2009. Acyl-CoA synthetase as a cancer survival factor: its inhibition enhances the efficacy of etoposide. Cancer Sci 100:1556-1562. 
Mazmanian SK, Liu CH, Tzianabos AO, and Kasper DL. 2005. An immunomodulatory molecule of symbiotic bacteria directs maturation of the host immune system. Cell 122:107-118.

Mukherji A, Kobiita A, Ye T, and Chambon P. 2013. Homeostasis in intestinal epithelium is orchestrated by the circadian clock and microbiota cues transduced by TLRs. Cell 153:812-827.

Myers CL, Robson D, Wible A, Hibbs MA, Chiriac C, Theesfeld CL, Dolinski K, and Troyanskaya OG. 2005. Discovery of biological networks from diverse functional genomic data. Genome Biol 6:R114.

Myers CL, and Troyanskaya OG. 2007. Context-sensitive data integration and prediction of biological networks. Bioinformatics 23:2322-2330.

Nephew KP, Polek TC, and Khan SA. 1996. Tamoxifen-induced proto-oncogene expression persists in uterine endometrial epithelium. Endocrinology 137:219-224.

Noble WS. 2009. A quick guide to organizing computational biology projects. PLoS Comput Biol 5:e1000424.

Oberti JM, Breser ML, Nuñez N, Maccioni M, Rodriguez N, Wantia N, Ertl T, Miethke T, and Rivero VE. 2011. Chemokine response induced by Chlamydia trachomatis in prostate derived CD45+ and CD45- cells. Reproduction 142:427-437.

Orchard S, Ammari M, Aranda B, Breuza L, Briganti L, Broackes-Carter F, Campbell NH, Chavali G, Chen C, delToro N, Duesbury M, Dumousseau M, Galeota E, Hinz U, lannuccelli M, Jagannathan S, Jimenez R, Khadake J, Lagreid A, Licata L, Lovering RC, Meldal B, Melidoni AN, Milagros M, Peluso D, Perfetto L, Porras P, Raghunath A, Ricard-Blum S, Roechert B, Stutz A, Tognolli M, van Roey K, Cesareni G, and Hermjakob H. 2014. The MIntAct project--IntAct as a common curation platform for 11 molecular interaction databases. Nucleic Acids Res 42:D358-363.

Pallen MJ, Chaudhuri RR, and Henderson IR. 2003. Genomic analysis of secretion systems. Curr Opin Microbiol 6:519-527.

Pei Z, Fraisl P, Shi X, Gabrielson E, Forss-Petter S, Berger J, and Watkins PA. 2013. Very long-chain acyl-CoA synthetase 3: overexpression and growth dependence in lung cancer. PLoS One 8:e69392.

Pei Z, Sun P, Huang P, Lal B, Laterra J, and Watkins PA. 2009. Acyl-CoA synthetase VL3 knockdown inhibits human glioma cell proliferation and tumorigenicity. Cancer Res 69:9175-9182.

Perkins ND. 2012. The diverse and complex roles of NF-kappaB subunits in cancer. Nat Rev Cancer 12:121-132.

Potti A, Dressman HK, Bild A, Riedel RF, Chan G, Sayer R, Cragun J, Cottrill H, Kelley MJ, Petersen R, Harpole D, Marks J, Berchuck A, Ginsburg GS, Febbo P, Lancaster J, and Nevins JR. 2006. Genomic signatures to guide the use of chemotherapeutics. Nat Med 12:1294-1300.

Pringle LM, Young R, Quick L, Riquelme DN, Oliveira AM, May MJ, and Chou MM. 2012. Atypical mechanism of NF-kappaB activation by TRE17/ubiquitin-specific protease 6 (USP6) oncogene and its requirement in tumorigenesis. Oncogene 31:3525-3535.

Pruitt KD, Tatusova T, and Maglott DR. 2005. NCBI Reference Sequence (RefSeq): a curated non-redundant sequence database of genomes, transcripts and proteins. Nucleic Acids Res 33:D501-504.

Rakhshandehroo M, Knoch B, Muller M, and Kersten S. 2010. Peroxisome proliferator-activated receptor alpha target genes. PPAR Res 2010.

Rao J, DiGiandomenico A, Unger J, Bao Y, Polanowska-Grabowska RK, and Goldberg JB. 2008. A novel oxidized low-density lipoprotein-binding protein from Pseudomonas aeruginosa. Microbiology 154:654-665.

Re A, Workman CT, Waldron L, Quattrone A, and Brunak S. 2014. Lineage-specific interface proteins match up the cell cycle and differentiation in embryo stem cells. Stem Cell Res 13:316-328.

Rhodes DR, Yu J, Shanker K, Deshpande N, Varambally R, Ghosh D, Barrette T, Pandey A, and Chinnaiyan AM. 2004. ONCOMINE: a cancer microarray database and integrated data-mining platform. Neoplasia 6:1-6.

Riester M, Wei W, Waldron L, Culhane AC, Trippa L, Oliva E, Kim SH, Michor F, Huttenhower C, Parmigiani G, and Birrer MJ. 2014. Risk prediction for late-stage ovarian cancer by meta-analysis of 1525 patient samples. J Natl Cancer Inst 106.

Rifai N, Gillette MA, and Carr SA. 2006. Protein biomarker discovery and validation: the long and uncertain path to clinical utility. Nat Biotechnol 24:971-983. 
1023

1024

1025

1026

1027

1028

1029

1030

1031

1032

1033

1034

1035

1036

1037

1038

1039

1040

1041

1042

1043

1044

1045

1046

1047

1048

1049

1050

1051

1052

1053

1054

1055

1056

1057

1058

1059

1060

1061

1062

1063

1064

1065

1066

1067

1068

1069

1070

Rocca-Serra P, Brandizi M, Maguire E, Sklyar N, Taylor C, Begley K, Field D, Harris S, Hide W, Hofmann O, Neumann S, Sterk P, Tong W, and Sansone SA. 2010. ISA software suite: supporting standardscompliant experimental annotation and enabling curation at the community level. Bioinformatics 26:2354-2356.

Round JL, and Mazmanian SK. 2009. The gut microbiota shapes intestinal immune responses during health and disease. Nature reviews Immunology 9:313-323.

Rustici G, Kolesnikov N, Brandizi M, Burdett T, Dylag M, Emam I, Farne A, Hastings E, Ison J, Keays M, Kurbatova N, Malone J, Mani R, Mupo A, Pedro Pereira R, Pilicheva E, Rung J, Sharma A, Tang YA, Ternent T, Tikhonov A, Welter D, Williams E, Brazma A, Parkinson H, and Sarkans U. 2013. ArrayExpress update-trends in database growth and links to data analysis tools. Nucleic Acids Res 41:D987-990.

Samuel Reich E. 2011. Cancer trial errors revealed. Nature 469:139-140.

Sandkvist M. 2001. Biology of type II secretion. Molecular microbiology 40:271-283.

Sayers EW, Barrett T, Benson DA, Bryant SH, Canese K, Chetvernin V, Church DM, DiCuccio M, Edgar R, Federhen S, Feolo M, Geer LY, Helmberg W, Kapustin Y, Landsman D, Lipman DJ, Madden TL, Maglott DR, Miller V, Mizrachi I, Ostell J, Pruitt KD, Schuler GD, Sequeira E, Sherry ST, Shumway M, Sirotkin K, Souvorov A, Starchenko G, Tatusova TA, Wagner L, Yaschenko E, and Ye J. 2009. Database resources of the National Center for Biotechnology Information. Nucleic Acids Res 37:D5-15.

Schulert GS, Feltman H, Rabin SD, Martin CG, Battle SE, Rello J, and Hauser AR. 2003. Secretion of the toxin ExoU is a marker for highly virulent Pseudomonas aeruginosa isolates obtained from patients with hospital-acquired pneumonia. J Infect Dis 188:1695-1706.

Seigne C, Fontaniere S, Carreira C, Lu J, Tong WM, Fontaniere B, Wang ZQ, Zhang CX, and Frappart L. 2010. Characterisation of prostate cancer lesions in heterozygous Men1 mutant mice. BMC Cancer 10:395.

Sharma VK, Sacco RE, Kunkle RA, Bearson SM, and Palmquist DE. 2012. Correlating levels of type III secretion and secreted proteins with fecal shedding of Escherichia coli O157:H7 in cattle. Infect Immun 80:13331342.

Silverman JM, Brunet YR, Cascales E, and Mougous JD. 2012. Structure and regulation of the type VI secretion system. Annual review of microbiology 66:453-472.

Sims AH. 2009. Bioinformatics and breast cancer: what can high-throughput genomic approaches actually tell us? J Clin Pathol 62:879-885.

Smith GK. 2005. Limma: linear models for microarray data. In: 'Bioinformatics and Computational Biology Solutions using R and Bioconductor'. R. Gentleman, V. Carey, S. Dudoit, R. Irizarry, W. Huber (eds). Springer:397-420.

Soupene E, Rothschild J, Kuypers FA, and Dean D. 2012. Eukaryotic Protein Recruitment into the Chlamydia Inclusion: Implications for Survival and Growth. PLoS One 7:e36843.

Staff S. 2011. Dealing with data. Challenges and opportunities. Introduction. Science 331:692-693.

Stark C, Breitkreutz BJ, Chatr-Aryamontri A, Boucher L, Oughtred R, Livstone MS, Nixon J, Van Auken K, Wang X, Shi X, Reguly T, Rust JM, Winter A, Dolinski K, and Tyers M. 2011. The BioGRID Interaction Database: 2011 update. Nucleic Acids Res 39:D698-704.

Su C, Peregrin-Alvarez JM, Butland G, Phanse S, Fong V, Emili A, and Parkinson J. 2008. Bacteriome.org--an integrated protein interaction database for E. coli. Nucleic Acids Res 36:D632-636.

Subramanian A, Tamayo P, Mootha VK, Mukherjee S, Ebert BL, Gillette MA, Paulovich A, Pomeroy SL, Golub TR, Lander ES, and Mesirov JP. 2005. Gene set enrichment analysis: a knowledge-based approach for interpreting genome-wide expression profiles. Proc Natl Acad Sci U S A 102:15545-15550.

Sun Y, Karmakar M, Taylor PR, Rietsch A, and Pearlman E. 2012. ExoS and ExoT ADP ribosyltransferase activities mediate Pseudomonas aeruginosa keratitis by promoting neutrophil apoptosis and bacterial survival. $J$ Immunol 188:1884-1895.

Suzek BE, Huang H, McGarvey P, Mazumder R, and Wu CH. 2007. UniRef: comprehensive and non-redundant UniProt reference clusters. Bioinformatics 23:1282-1288. 
Szklarczyk D, Franceschini A, Kuhn M, Simonovic M, Roth A, Minguez P, Doerks T, Stark M, Muller J, Bork P, Jensen LJ, and von Mering C. 2011. The STRING database in 2011: functional interaction networks of proteins, globally integrated and scored. Nucleic Acids Res 39:D561-568.

Taminau J, Steenhoff D, Coletta A, Meganck S, Lazar C, de Schaetzen V, Duque R, Molter C, Bersini H, Nowe A, and Weiss Solis DY. 2011. inSilicoDb: an R/Bioconductor package for accessing human Affymetrix expert-curated datasets from GEO. Bioinformatics 27:3204-3205.

Tremaroli V, and Backhed F. 2012. Functional interactions between the gut microbiota and host metabolism. Nature 489:242-249.

Tseng TT, Tyler BM, and Setubal JC. 2009. Protein secretion systems in bacterial-host associations, and their description in the Gene Ontology. BMC Microbio/ 9 Suppl 1:S2.

van 't Veer LJ, Dai H, van de Vijver MJ, He YD, Hart AA, Mao M, Peterse HL, van der Kooy K, Marton MJ, Witteveen AT, Schreiber GJ, Kerkhoven RM, Roberts C, Linsley PS, Bernards R, and Friend SH. 2002. Gene expression profiling predicts clinical outcome of breast cancer. Nature 415:530-536.

van lersel MP, Pico AR, Kelder T, Gao J, Ho I, Hanspers K, Conklin BR, and Evelo CT. 2010. The BridgeDb framework: standardized access to gene, protein and metabolite identifier mapping services. $B M C$ Bioinformatics 11:5.

Viechtbauer W. 2010. Conducting meta-analyses in $\{R\}$ with the $\{$ metafor $\}$ package. Journal of Statistical Software 36:1-48.

Waldron L, Coller HA, and Huttenhower C. 2012. Integrative approaches for microarray data analysis. Methods Mol Biol 802:157-182.

Waldron L, Haibe-Kains B, Culhane AC, Riester M, Ding J, Wang XV, Ahmadifar M, Tyekucheva S, Bernau C, Risch T, Ganzfried BF, Huttenhower C, Birrer M, and Parmigiani G. 2014. Comparative meta-analysis of prognostic gene signatures for late-stage ovarian cancer. J Nat/ Cancer Inst 106.

Warde-Farley D, Donaldson SL, Comes O, Zuberi K, Badrawi R, Chao P, Franz M, Grouios C, Kazi F, Lopes CT, Maitland A, Mostafavi S, Montojo J, Shao Q, Wright G, Bader GD, and Morris Q. 2010. The GeneMANIA prediction server: biological network integration for gene prioritization and predicting gene function. Nucleic Acids Res 38:W214-220.

Wassink I, Rauwerda H, Neerincx PB, van der Vet PE, Breit TM, Leunissen JA, and Nijholt A. 2009. Using R in Taverna: RShell v1.2. BMC Res Notes 2:138.

Wen Y, Yan DH, Spohn B, Deng J, Lin SY, and Hung MC. 2000. Tumor suppression and sensitization to tumor necrosis factor alpha-induced apoptosis by an interferon-inducible protein, p202, in breast cancer cells. Cancer Res 60:42-46.

Williamson KS, Richards LA, Perez-Osorio AC, Pitts B, McInnerney K, Stewart PS, and Franklin MJ. 2012. Heterogeneity in Pseudomonas aeruginosa biofilms includes expression of ribosome hibernation factors in the antibiotic-tolerant subpopulation and hypoxia-induced stress response in the metabolically active population. J Bacteriol 194:2062-2073.

Wilson G, Aruliah DA, Brown CT, Chue Hong NP, Davis M, Guy RT, Haddock SH, Huff KD, Mitchell IM, Plumbley MD, Waugh B, White EP, and Wilson P. 2014. Best practices for scientific computing. PLoS Biol 12:e1001745.

Wirapati P, Sotiriou C, Kunkel S, Farmer P, Pradervand S, Haibe-Kains B, Desmedt C, Ignatiadis M, Sengstag T, Schutz F, Goldstein DR, Piccart M, and Delorenzi M. 2008. Meta-analysis of gene expression profiles in breast cancer: toward a unified understanding of breast cancer subtyping and prognosis signatures. Breast Cancer Res 10:R65.

Wu CJ, Conze DB, Li T, Srinivasula SM, and Ashwell JD. 2006. Sensing of Lys 63-linked polyubiquitination by NEMO is a key event in NF-kappaB activation [corrected]. Nat Cell Biol 8:398-406.

Zhang L, Hinz AJ, Nadeau JP, and Mah TF. 2011. Pseudomonas aeruginosa tssC1 links type VI secretion and biofilm-specific antibiotic resistance. J Bacteriol 193:5510-5513. 
Zhu Y, and Xu W. 2008. EzArray: a web-based highly automated Affymetrix expression array data management and analysis system. BMC Bioinformatics 9:46.

Zuberi K, Franz M, Rodriguez H, Montojo J, Lopes CT, Bader GD, and Morris Q. 2013. GeneMANIA prediction server 2013 update. Nucleic Acids Res 41:W115-122. 
1152 provided for the user as text files, while all expression data and associated metadata are saved as 1153 individual text files and as an $\mathrm{R}$ data file. The bottom panel illustrates how generated data can be 1154 integrated by subsequent network (see prostate cancer and bacterial studies) or expression (see murine 1155 differential gene expression) meta-analysis. For example, network integration is a convenient way to 1156 combines multiple datasets of different types and sources, such as co-expression, physical gene 1157 interactions, regulatory interactions, or posttranslational modification, into one functional network.

1158 Figure 2: MEN1 and ACBD6 associated with the NFKB signaling pathway in human prostate 1159 cancer. High confidence subgraph extracted from a functional network integrating ten prostate cancer 1160 specific gene expression data sets from GEO (Supplementary Table 1). This subnetwork was 1161 generated using a seed gene set of ten genes from the NFkB signaling pathway in BioCarta (blue 1162 circles). Nine genes (black circles) were immediately recovered that are also known to be involved in $1163 \mathrm{Nf \kappa B}$ signaling. Additional genes represent candidates implicated in NFkB involvement during 1164 prostate cancer, in particular MEN1 and ACBD6.

1165

1166

1167

1168

1169

1170

1171

1172

1173

1174

1175

1176

1177

1178

1179

1180

1181

1182

1183

1184

1185

1186

1187

1188

1189

Figure 3: Differential expression meta-analysis of germ-free versus conventional mice. ARepA metadata allowed the identification of six murine gene expression datasets with intestinal tissue from paired germ-free and conventional mice (Supplementary Table 2). The automatically generated R expression sets were meta-analyzed using R/limma (Smith 2005) and R/metafor (Viechtbauer 2010) through a random-effects model, revealing the Ppar- $\alpha$ signaling pathway as one of several differentially regulated gene sets. In (A) the fold changes are presented for all significantly differentially expressed genes from this pathway in individual datasets, and $(B / C)$ show the corresponding forest plots for the Ppar- $\alpha$ and Rxr- $\alpha$ genes, which are consistently upregulated in these datasets.

Figure 4: Integrated molecular networks for comparative microbial functional genomics. ARepA allowed the retrieval of standardized gene expression and interaction data for three microbial species based on a shared gene identifier to assess functional differences in conserved and non-conserved secretion pathways. High-confidence subgraphs were extracted from species-specific integrated functional networks around genes from species-specific secretion pathways to identify highly functionally related gene clusters within each individual system. These subgraphs represent gene clusters of Sec and Tat genes in B. subtilis (A), sec, tat, and Type II genes in E. coli (B), and sec, tat, Type II, Type III, and Type VI genes in P. Aeruginosa (C). From each of these species-specific molecular networks we recovered highly functionally related gene clusters and conserved and nonconserved components from the peptide secretion system.

Figure 5: Analysis and processing steps available for datasets from each data source. The main steps of ARepA are divided up into four components: (1) Configuration and data integration: optional user-provided information can be merged with default data/metadata from the repositories. This allows, for example, integration of expert curated metadata with automatically annotated metadata. (2) Custom data processing, including the default and customizable gene mapping and metadata annotation, as well as processes for file format detection and conversion. (3) Data normalization: gene identifiers are standardized, gene expression levels are normalized (e.g. log-transformed), missing values are imputed 
1190

1191

1192

1193

1194

1195

1196

1197

1198

1199

1200

1201

1202

1203

1204

1205

1206

1207

1208

1209

1210

1211

1212

1213

1214

1215

1216

1217

1218

1219

1220

1221

using k-nearest neighborhoods, and duplicate entries are merged. (4) Data export: data file formats are normalized to tab-delimited text, and co-expression networks in text and binary formats are constructed. Gene expression datasets and automatically generated documentation are further compiled into an $\mathrm{R}$ data file.

(1)

(1)

(1)

(1) 
1222 expression for all genes between germ-free gut and wild type gut within each dataset using the $1223 \mathrm{R} /$ limma package. Next, we performed a meta-analysis using the $\mathrm{R} /$ metafor package on these six 1224 datasets by applying a random-effects model on the fold changes, fitting the model with the restricted 1225 maximum-likelihood estimator (REML). We finally tested all genes and their resulting meta-p-values 1226 for gene set enrichment in KEGG and BioCarta pathways which are shown in this table.

1227 Supplementary Table 4: Lists of query genes for extracting high-confidence subgraphs in human 1228 and bacteria. To extract high-confidence subgraphs from global networks, we defined lists of query 1229 genes for each context. First, we defined a list of 12 query genes from the NFkB signaling pathway in 1230 BioCarta for extracting a high-confidence subgraph from the prostate cancer specific co-expression 1231 network in human. Second, we defined three lists of query gene sets of the sec and tat genes and genes 1232 from the Type II, Type III, and Type VI secretion systems for one Gram-positive (B. subtilis) and two 1233 Gram-positive bacteria (E. coli and P. aeruginosa).

1234 Supplementary Table 5: Meta-analysis of murine gene expression datasets from germ-free vs. 1235 wild type intestinal tissue. We conducted a differential expression meta-analysis of genes and 1236 pathways up- or down-regulated in the germ-free murine gut (Supplementary Figure 1), using six 1237 case-control datasets (Supplementary Table 2). We first computed differential expression for all 1238 genes between germ-free gut and wild type gut within each dataset using the R/limma package. Next, 1239 we performed a meta-analysis using the R/metafor package on these six datasets by applying a random1240 effects model on the fold changes, fitting the model with the restricted maximum-likelihood estimator 1241 (REML).

\section{Supplementary Text 1: Details of ARepA case studies.}

\section{Supplementary Text 2: Metadata file example.}


ARepA is an extensible, modular Automated Repository Acquisition system for reproducible biological data acquisition and processing.

ARepA is a framework for reproducible biological data mining and analysis. It can retrieve heterogeneous data from multiple public repositories in a uniform environment and format, currently allowing configurable data access for any organism(s) to the Gene Expression Omnibus (GEO) ( Barrett et al. 2011 ), IntAct ( Kerrien et al. 2012 ) , BioGRID ( Stark et al. 2011 ) , RegulonDB ( Gama-Castro et al. 2011 ) , STRING ( Szklarczyk et al. 2011 ), Bacteriome ( Su et al. 2008 ), and MPIDB ( Goll et al. 2008 ) databases. Using ARepA includes the following steps: (i) user input, (ii) data processing, and (iii) output formatting. The input phase is the only aspect of the ARepA pipeline that requires direct user oversight. The user's input to ARepA can be as simple a list of organisms-of-interest; ARepA then uses this list as a query for recovering interactome network and gene expression data specific to those organisms. Advanced users also have the option of providing custom gene mapping files, metadata, and/or normalization schemes, as well as fine-tuning the list of data sources to be searched. The data processing phase is divided into a series of automated steps in which raw interactome network and gene expression data are downloaded, converted to a common gene-naming scheme, and normalized for between-dataset comparison. During this phase, integrated gene expression data are analyzed for co-expression relationships, which contributes an additional co-expression network to the final network output. All network data are provided for the user as text files, while all expression data and associated metadata are saved as individual text files and as an $\mathrm{R}$ data file. The bottom panel illustrates how generated data can be integrated by subsequent network (see prostate cancer and bacterial studies) or expression (see murine differential gene expression) meta-analysis. For example, network integration is a convenient way to combines multiple datasets of different types and sources, such as co-expression, physical gene interactions, regulatory interactions, or 


\section{posttranslational modification, into one functional network.}

\section{ARepA: Automated Repository Acquisition}

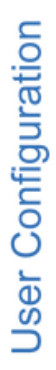
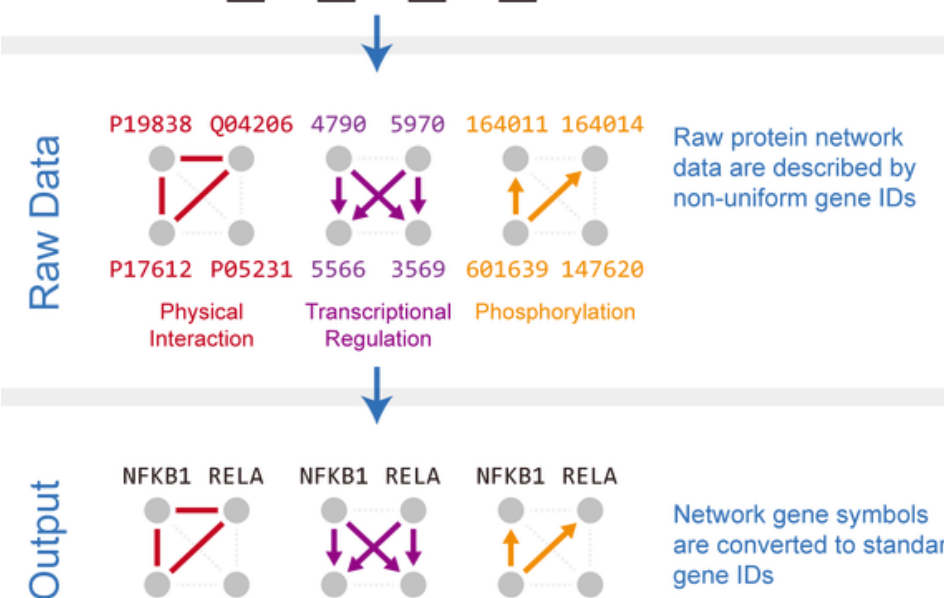

NFKB1 RELA

PRKACA IL

PRKACA IL

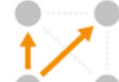

Network gene symbols are converted to standard gene IDs

NFKB1 RELA

:

A co-expression network is generated from the processed gene expression data (right)

PRKACA IL6
User-selected protein network data are downloaded from a variety of databases

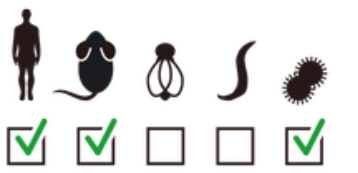

Basic Configuration

the user specifies a list of organisms to use as a query for collecting protein network and gene expression data
Additional Options

The user can also provide:

- custom gene mappings

- custom metadata

- custom normalization scheme

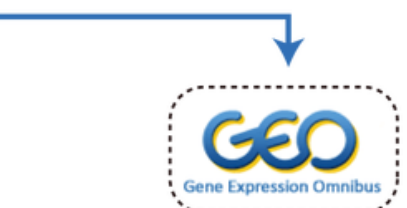

User-selected gene expression data and related metadata are downloaded from the GEO database
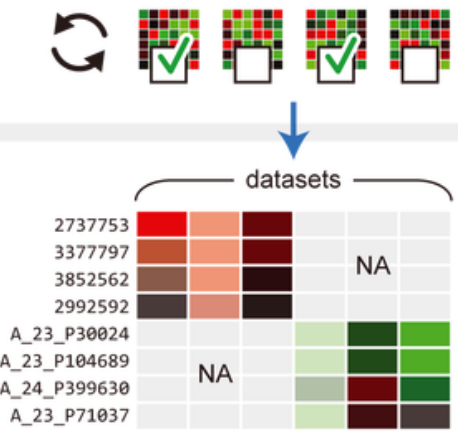

Raw gene expression data are described by non-uniform gene IDs and vary in average intensity

A_24_P399630

A_23_P71037

NA

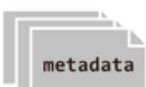

\section{metadata}
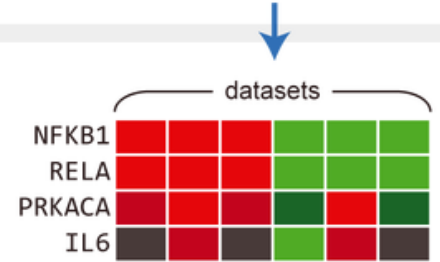

Probe sets are collapsed to standard gene IDs; datasets are normalized to be comparable

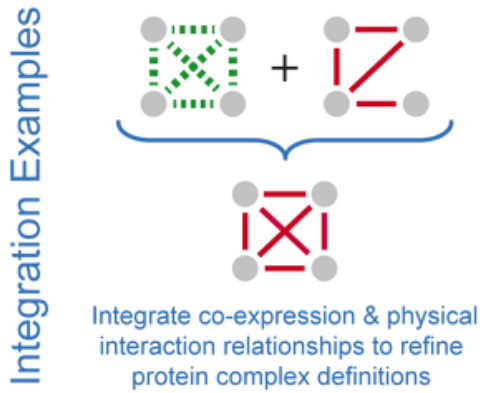
protein complex definitions

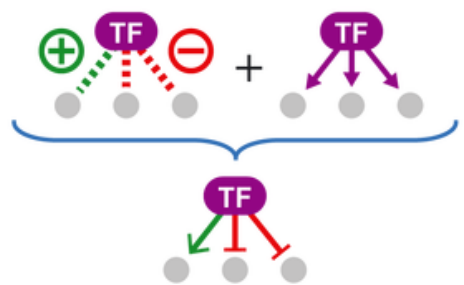

Integrate co-expression \& transcription factor (TF) binding relationships to predict activation vs. suppression of expression
Processed expression data and all metadata are saved as an $\mathrm{R}$ package 
2

MEN1 and ACBD6 associated with the NFKB signaling pathway in human prostate cancer.

High confidence subgraph extracted from a functional network integrating ten prostate cancer specific gene expression data sets from GEO (Supplementary Table 1). This subnetwork was generated using a seed gene set of ten genes from the NFKB signaling pathway in BioCarta (blue circles). Nine genes (black circles) were immediately recovered that are also known to be involved in NfKB signaling. Additional genes represent candidates implicated in NFKB involvement during prostate cancer, in particular MEN1 and ACBD6. 


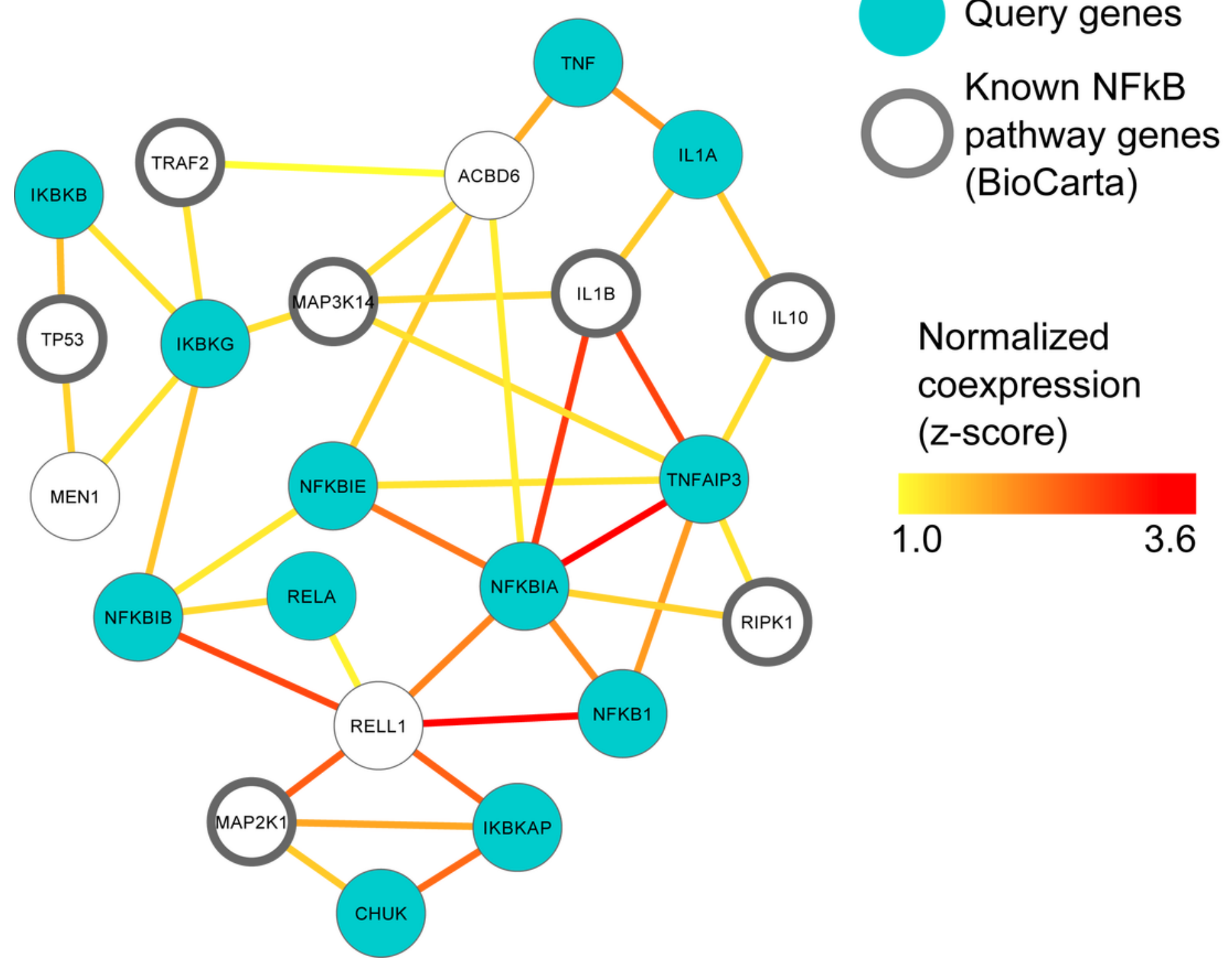


Differential expression meta-analysis of germ-free versus conventional mice.

ARepA metadata allowed the identification of six murine gene expression datasets with intestinal tissue from paired germ-free and conventional mice (Supplementary Table 2). The automatically generated R expression sets were meta-analyzed using R/limma ( Smith 2005 ) and R/metafor ( Viechtbauer 2010 ) through a random-effects model, revealing the Ppar- $\alpha$ signaling pathway as one of several differentially regulated gene sets. In (A) the fold changes are presented for all significantly differentially expressed genes from this pathway in individual datasets, and $(B / C)$ show the corresponding forest plots for the Ppar- $\alpha$ and Rxr- $\alpha$ genes, which are consistently upregulated in these datasets.

A

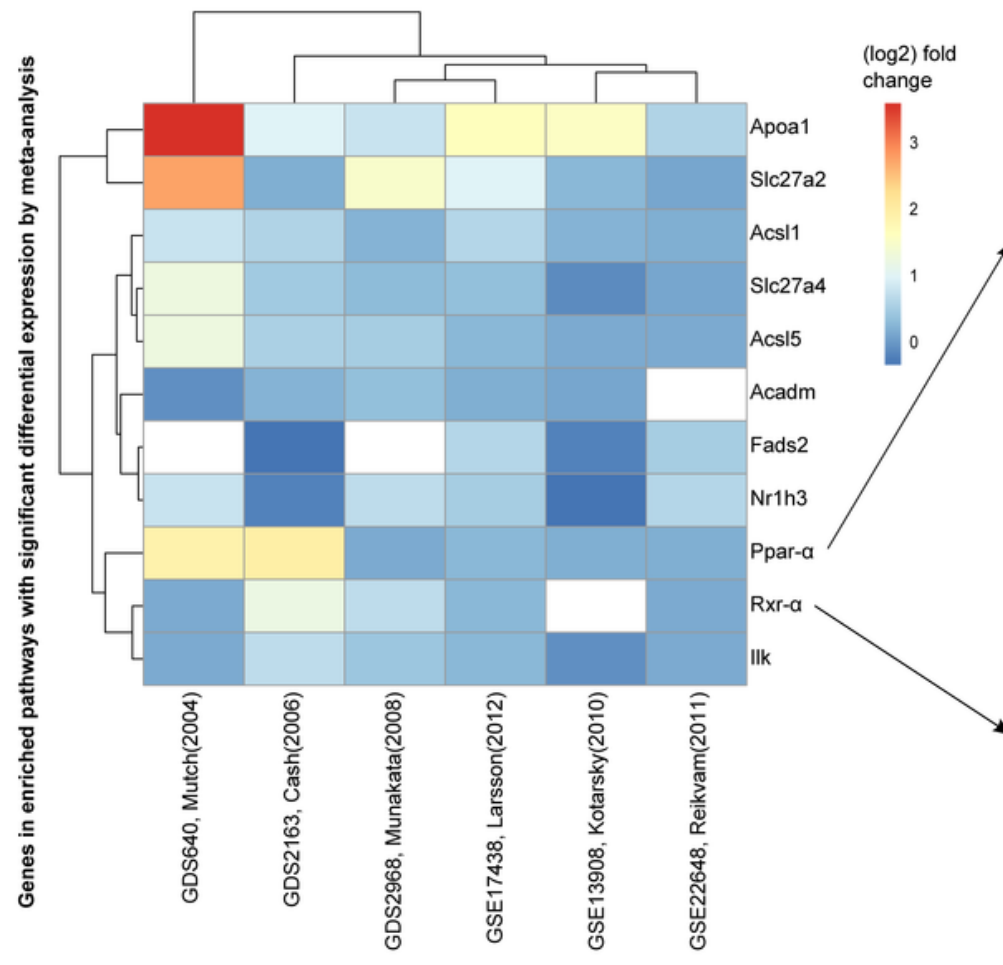

Gene expression datasets assessing germ free murine gut tissue
B

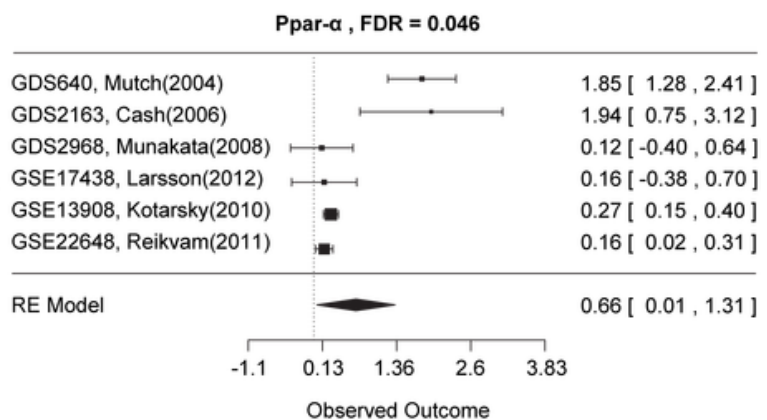

C

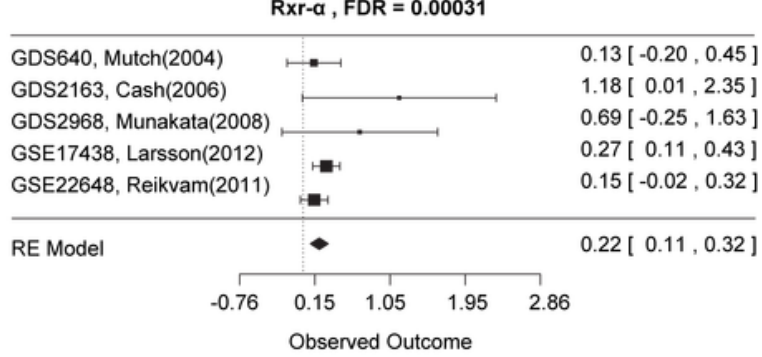


Integrated molecular networks for comparative microbial functional genomics.

ARepA allowed the retrieval of standardized gene expression and interaction data for three microbial species based on a shared gene identifier to assess functional differences in conserved and non-conserved secretion pathways. High-confidence subgraphs were extracted from species-specific integrated functional networks around genes from speciesspecific secretion pathways to identify highly functionally related gene clusters within each individual system. These subgraphs represent gene clusters of Sec and Tat genes in $B$. subtilis (A), sec, tat, and Type II genes in E. coli (B), and sec, tat, Type II, Type III, and Type VI genes in $P$. Aeruginosa (C). From each of these species-specific molecular networks we recovered highly functionally related gene clusters and conserved and non-conserved components from the peptide secretion system.
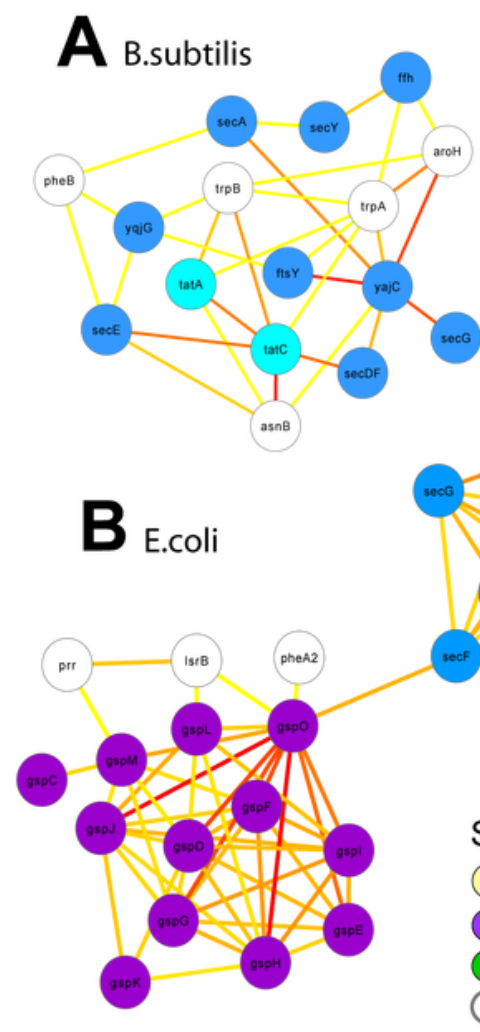
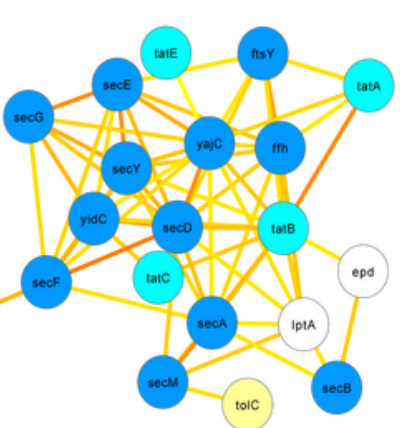

Secretion type type I

type II type III predicted type III
C P.aeruginosa
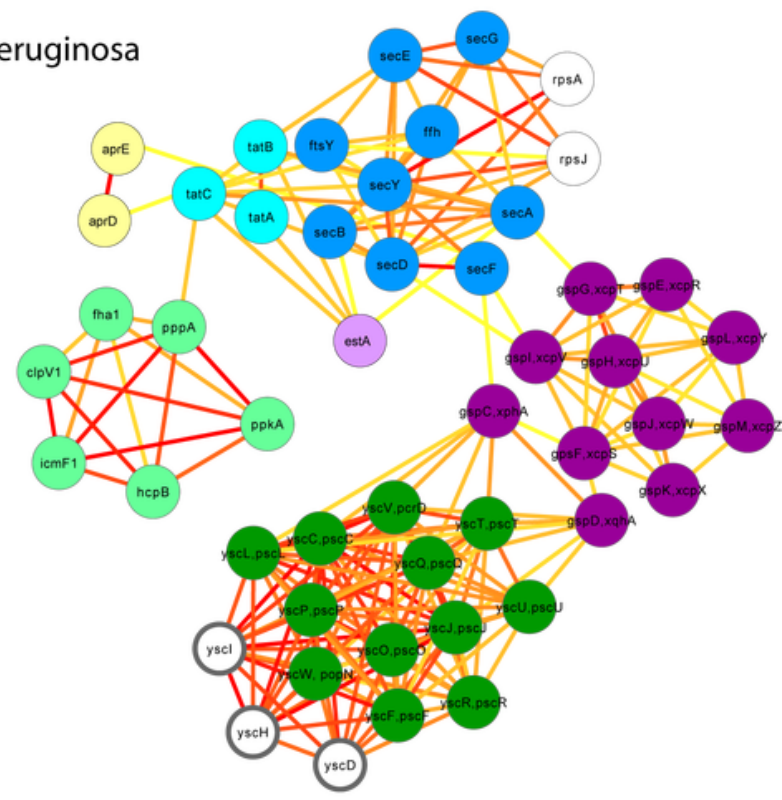

type V

type VI

sec

tat
Normalized coexpression (z-score)

1.0 


\section{5}

Analysis and processing steps available for datasets from each data source.

The main steps of ARepA are divided up into four components: (1) Configuration and data integration: optional user-provided information can be merged with default data/metadata from the repositories. This allows, for example, integration of expert curated metadata with automatically annotated metadata.(2) Custom data processing, including the default and customizable gene mapping and metadata annotation, as well as processes for file format detection and conversion. (3) Data normalization: gene identifiers are standardized, gene expression levels are normalized (e.g. log-transformed), missing values are imputed using knearest neighborhoods, and duplicate entries are merged.(4) Data export: data file formats are normalized to tab-delimited text, and co-expression networks in text and binary formats are constructed. Gene expression datasets and automatically generated documentation are further compiled into an R data file.<!---u-->

ARepA: Data Acquisition Per Repository

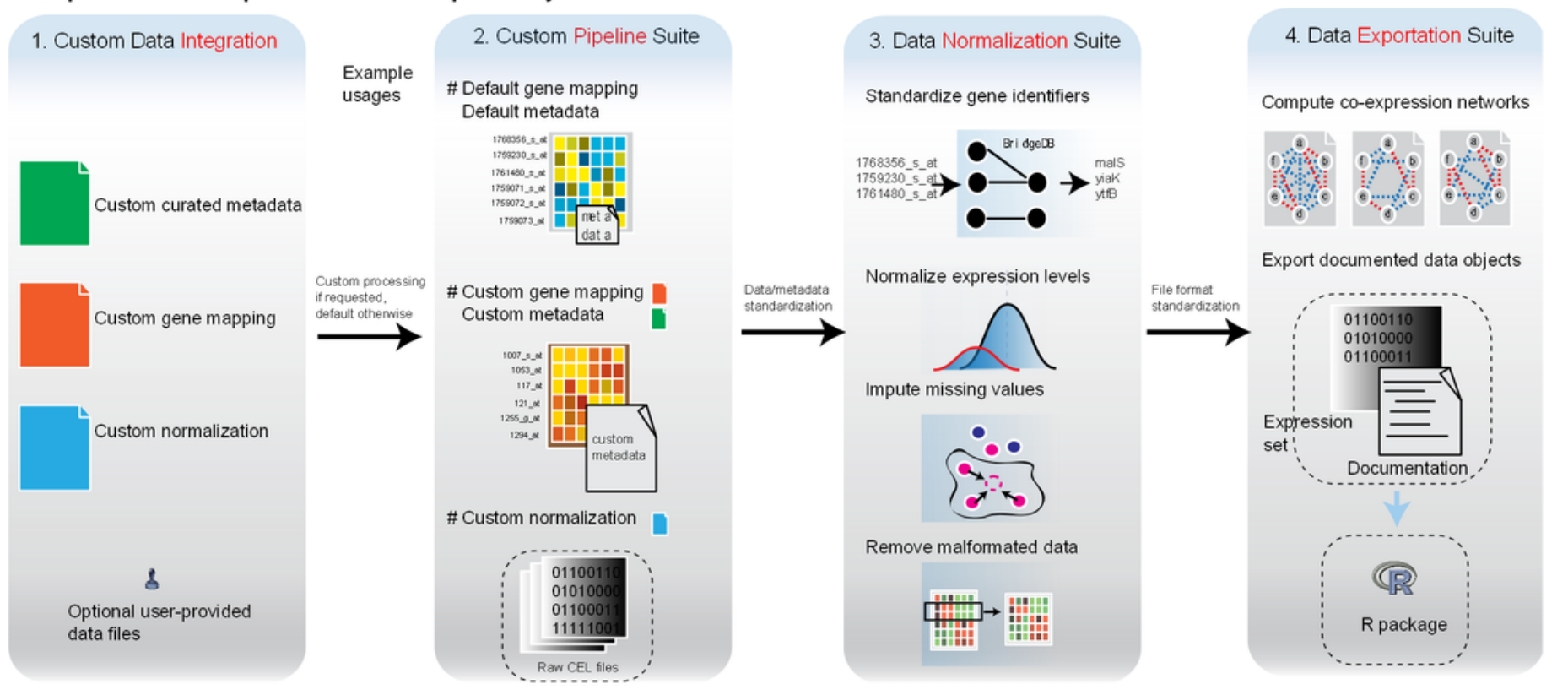


Table $\mathbf{1}_{\text {(on next page) }}$

ARepA extracts heterogeneous data from several distinct data types.

ARepA extracts heterogeneous data from several distinct data types: physical interactions (Bacteriome, BioGRID, MPIDB, and IntAct), functional associations (STRING), gene expression (GEO), and regulatory interactions (RegulonDB). All repositories can be configured to retrieve only a specified range of taxonomic identifiers, and individual dataset IDs can be included or excluded from applicable repositories. Output data includes standardized data files in multiple formats (text tables, interaction networks, and/or R packages) as well as metadata for all data types and datasets. 
2 Table 1: ARepA extracts heterogeneous data from several distinct data types: physical 3 interactions (Bacteriome, BioGRID, MPIDB, and IntAct), functional associations (STRING), 4 gene expression (GEO), and regulatory interactions (RegulonDB). All repositories can be 5 configured to retrieve only a specified range of taxonomic identifiers, and individual dataset IDs can be 6 included or excluded from applicable repositories. Output data includes standardized data files in

7 multiple formats (text tables, interaction networks, and/or R data files) as well as metadata for all data 8 types and datasets.

\begin{tabular}{|c|c|c|c|c|c|}
\hline Database & $\begin{array}{l}\text { Interaction } \\
\text { data type }\end{array}$ & $\begin{array}{l}\# \quad \text { of } \\
\text { species } \\
\left(\begin{array}{l}\text { as of } \\
03 / 2013)\end{array}\right.\end{array}$ & $\begin{array}{ll}\# & \text { of } \\
\text { interactions } \\
\text { (as of of } \\
\text { 03/2013) }\end{array}$ & Reference & $\begin{array}{l}\text { Metadata output } \\
\text { type }\end{array}$ \\
\hline Bacteriome & Physical & 1 & 3,888 & $\frac{(\mathrm{Su} \text { et al. }}{2008)}$ & taxonomy ID \\
\hline BioGRID & Physical & 32 & 349,696 & $\frac{(\text { Stark et al. }}{\underline{2011)}}$ & $\begin{array}{l}\text { platform, } \\
\text { experiment type, } \\
\text { taxonomy ID }\end{array}$ \\
\hline IntAct & Physical & 278 & 239,940 & $\begin{array}{l}\frac{(\text { Kerrien et }}{\text { al. 2012; }} \\
\text { Orchard et } \\
\underline{\text { al. 2014) }}\end{array}$ & $\begin{array}{l}\text { platform, } \\
\text { experiment type, } \\
\text { taxonomy ID }\end{array}$ \\
\hline MPIDB & Physical & 250 & 24,295 & $\frac{\text { (Goll et al. }}{\underline{2008)}}$ & $\begin{array}{l}\text { platform, } \\
\text { experiment type, } \\
\text { taxonomy ID }\end{array}$ \\
\hline RegulonDB & regulatory & 1 & 4,096 & $\frac{\frac{(\text { Gama- }}{\text { Castro }}}{\text { al. 2011) }}$ & taxonomy ID \\
\hline STRING & functional & 1,133 & $1,640,707$ & $\frac{\overline{\text { (Szklarczyk }}}{\text { et al. 2011) }}$ & $\begin{array}{l}\text { taxonomy ID, } \\
\text { mode }\end{array}$ \\
\hline GEO & expression & 1,967 & & 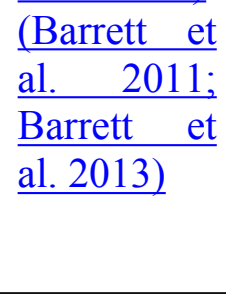 & $\begin{array}{lr}\text { taxonomy } & \text { ID, } \\
\text { experiment } & \text { type, } \\
\text { pubmed } & \text { ID, } \\
\text { platform, title, } \\
\text { glossary, channels, } \\
\text { conditions }\end{array}$ \\
\hline
\end{tabular}


Table 2 (on next page)

ARepA currently yields more than $2.7 \mathrm{~K}$ expression data sets from GEO and more than $2 \mathrm{M}$ interactions.

A: ARepA currently yields more than $2.7 \mathrm{~K}$ expression data sets from GEO covering more than $800 \mathrm{~K}$ samples. B: ARepA currently yields more than $2 \mathrm{M}$ interactions: $600 \mathrm{~K}$ physical interactions (Bacteriome, BioGRID, IntAct, and MPIDB), 4K regulatory interactions (RegulonDB), and 1.5M functional associations (STRING). 
2 Table 2A: ARepA currently yields more than 2.7K expression data sets from GEO covering more

3 than $800 \mathrm{~K}$ samples (as of 03/2013) after standardization (UniRef).

\begin{tabular}{l|llllll} 
Datatype & Human & Mouse & Yeast & $\begin{array}{l}\text { E. } \\
\text { coli }\end{array}$ & $\begin{array}{l}\text { B. } \\
\text { subtilis }\end{array}$ & $\begin{array}{l}\text { P. } \\
\text { aeruginosa }\end{array}$ \\
\hline Number of datasets and samples & & & & \\
\hline $\begin{array}{l}\text { Expression } \\
\text { datasets }\end{array}$ & 10,575 & 3,940 & 162 & 184 & 15 & 73 \\
$\begin{array}{l}\text { Expression } \\
\text { samples/conditions }\end{array}$ & 467,369 & 57,813 & 2,374 & 1,955 & 199 & 802 \\
\hline
\end{tabular}

7 Table 2B: ARepA currently yields more than 2M interactions: $600 \mathrm{~K}$ physical interactions 8 (Bacteriome, BioGRID, IntAct, and MPIDB), 4K regulatory interactions (RegulonDB), and 1.5M 9 functional associations (STRING) (as of 03/2013) after standardization (UniRef).

\begin{tabular}{|c|c|c|c|c|c|c|c|}
\hline Datatype & Database & Human & Mouse & Yeast & E. coli & $\begin{array}{l}\text { B. } \\
\text { subtilis }\end{array}$ & $\begin{array}{l}P . \\
\text { aeruginosa }\end{array}$ \\
\hline \multicolumn{8}{|c|}{ Number of interactions } \\
\hline \multirow[t]{4}{*}{ Physical } & Bacteriome & na & na & na & 6,741 & na & na \\
\hline & BioGRID & 49,794 & 1,815 & 103,475 & 0 & 0 & 0 \\
\hline & IntAct & 46,961 & 1,025 & 30,205 & 10,992 & 407 & 2 \\
\hline & MPIDB & na & na & na & 1,449 & 118 & 20 \\
\hline Regulatory & RegulonDB & na & na & na & 71 & na & na \\
\hline Functional & STRING & 226,986 & 1,129 & 22,222 & 85,608 & 2,931 & 44,032 \\
\hline
\end{tabular}

